\title{
ARTICLE OPEN mTORC1 induces plasma membrane depolarization and promotes preosteoblast senescence by regulating the sodium channel Scnla
}

Ajuan Chen ${ }^{1}$, Jian Jin², Shasha Cheng ${ }^{3}$, Zezheng Liu', Cheng Yang ${ }^{1}$, Qingjing Chen ${ }^{1}$, Wenquan Liang (iD ${ }^{1}$, Kai Li $^{1}$, Dawei Kang ${ }^{1}$, Zhicong Ouyang ${ }^{1}$, Chenfeng Yao ${ }^{1}$, Xiaochun Bai $\mathbb{D}^{1,4}$, Qingchu $\mathrm{Li}^{1}$, Dadi Jin ${ }^{1 凶}$ and Bin Huang (D) $^{1 凶}$

Senescence impairs preosteoblast expansion and differentiation into functional osteoblasts, blunts their responses to bone formation-stimulating factors and stimulates their secretion of osteoclast-activating factors. Due to these adverse effects, preosteoblast senescence is a crucial target for the treatment of age-related bone loss; however, the underlying mechanism remains unclear. We found that mTORC1 accelerated preosteoblast senescence in vitro and in a mouse model. Mechanistically, mTORC1 induced a change in the membrane potential from polarization to depolarization, thus promoting cell senescence by increasing $\mathrm{Ca}^{2+}$ influx and activating downstream NFAT/ATF3/p53 signaling. We further identified the sodium channel Scn1a as a mediator of membrane depolarization in senescent preosteoblasts. Scn 1 a expression was found to be positively regulated by mTORC1 upstream of C/EBPa, whereas its permeability to $\mathrm{Na}^{+}$was found to be gated by protein kinase A (PKA)-induced phosphorylation. Prosenescent stresses increased the permeability of Scn 1 a to $\mathrm{Na}^{+}$by suppressing PKA activity and induced depolarization in preosteoblasts. Together, our findings identify a novel pathway involving mTORC1, Scn 1a expression and gating, plasma membrane depolarization, increased $\mathrm{Ca}^{2+}$ influx and NFAT/ATF3/p53 signaling in the regulation of preosteoblast senescence. Pharmaceutical studies of the related pathways and agents might lead to novel potential treatments for agerelated bone loss.

\section{INTRODUCTION}

Bone remodeling is mediated mainly by bone-resorbing osteoclasts and bone-forming osteoblasts and their precursors. In the first three decades of healthy human life, bone formation dominates until peak bone mass is achieved. ${ }^{1}$ With aging, bone remodeling is interrupted in favor of bone resorption, which causes bone loss and predisposes the skeleton to fractures. Recent studies have revealed a causal role of senescent cells in the formation-resorption shift in bone remodeling and age-related bone loss. Multiple cell types in the bone microenvironment become senescent with aging. ${ }^{2}$ Eliminating senescent cells, however, has both antiresorptive and anabolic effects on bone. ${ }^{3}$ Moreover, the pathophysiology of senile osteoporosis in aged humans is characterized by a decrease in bone formation that exceeds the decrease in bone resorption. ${ }^{4}$ Therefore, senescencerelated osteoblast dysfunction is the main cause of age-related bone loss. ${ }^{5}$

Bone formation requires an adequate number of mature osteoblastic cells, which are postmitotic cells that have a short lifespan and must be continually replaced with new cells. ${ }^{6}$ Osteoblasts arise from preosteoblasts, which express the transcription factors Osterix (Osx) and Runx2. ${ }^{7}$ However, senescence impairs preosteoblast proliferation and differentiation into mature osteoblasts. ${ }^{8}$ Senescent preosteoblasts also exhibit a blunted response to growth factors and hormones known to control bone formation. ${ }^{9-11}$ Moreover, these cells have been reported to create a defective microenvironment for bone formation by secreting several inflammatory and osteoclastactivating factors. $^{12}$ Due to these adverse effects, preosteoblast senescence is a crucial target for the treatment of age-related bone loss; however, the underlying mechanism remains unclear.

As the core signaling pathway in regulating lifespan, mTORC1 is associated with various processes related to aging, such as nutrient sensing, maintenance of proteostasis, autophagy, mitochondrial dysfunction and cellular senescence. ${ }^{13}$ Interestingly, emerging evidence suggests different requirements for mTORC1 in early life vs. late life. mTORC1 is needed for development and reproduction because its functional absence is lethal during embryogenesis. ${ }^{14}$ Later in life, however, active mTORC 1 drives senescence and increases the risk of disease. ${ }^{15}$ We previously reported essential roles of mTORC1 in preosteoblast expansion and function during bone development; ${ }^{16,17}$

\footnotetext{
${ }^{1}$ Academy of Orthopedics, Guangdong Province, Guangdong Provincial Key Laboratory of Bone and Joint Degeneration Diseases, Department of Spine Surgery, The Third Affiliated Hospital of Southern Medical University, Guangzhou, China; ${ }^{2}$ Department of Spine Surgery, Nanfang Hospital, Southern Medical University, Guangzhou, China;

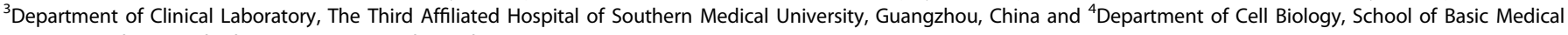
Science, Southern Medical University, Guangzhou, China

Correspondence: Dadi Jin (nyorthop@163.com) or Bin Huang (binxue483@163.com)

These authors contributed equally: Ajuan Chen, Jian Jin
}

Received: 8 April 2021 Revised: 16 November 2021 Accepted: 17 January 2022

Published online: 08 March 2022 
however, its roles in preosteoblast senescence and bone aging warrant further investigation.

Cells have a transmembrane potential, ${ }^{18}$ which is maintained by the balance between ions on both sides of the plasma membrane. Although the underlying mechanisms are unclear, membrane potential changes have been revealed to be involved in the senescence of fibroblasts ${ }^{19}$ and epithelial cells. ${ }^{20}$ Because mTORC1 has been reported to be associated with membrane depolarization, we investigated whether membrane depolarization has a role in mTORC1-regulated preosteoblast senescence and further unveiled the underlying mechanisms. Hence, the present study sheds new light on a novel electrophysiological pathway controlling preosteoblast senescence and bone aging.

\section{RESULTS}

mTORC1 is activated in senescent preosteoblasts

$\mathrm{We}^{17}$ and other groups $\mathrm{s}^{21,22}$ have reported that mTORC1 inactivation by deletion of Raptor (mTORC1-specific component) in preosteoblasts ( $\triangle$ Raptor) causes lower bone mass in young mice. Interestingly, these mice regained bone mass to an even greater extent than the littermate controls at an old age (18 months) (Fig. 1a, b), thus indicating the different roles of $\mathrm{MTORC} 1$ in preosteoblasts during bone aging versus development.

To determine the role of mTORC1 during preosteoblast senescence, we first examined mTORC1 levels in preosteoblasts during senescence. As reported previously, ${ }^{8}$ the numbers of both Osx-positive preosteoblasts (Fig. S1a) and their descendants, osteocalcin (Ocn)-positive osteoblasts (Fig. S1b), were lower in the bones of the old mice ( 18 months) than the young mice (9 months). Importantly, the senescent preosteoblasts in the old mice (Fig. 1c) showed significantly elevated mTORC1 activity, as revealed by the phosphorylation levels of S6 (Ser235/236) (Fig. 1d).

To confirm these results in vitro, we further induced senescence in the MC3T3-E1 preosteoblast cell line by using reactive oxygen species (ROS). ROS treatment increased senescence-associated$\beta$-galactosidase (SA- $\beta$-Gal) activity (Fig. 1e) and proliferative arrest (Fig. 1f) and elevated senescence marker (p16 and p53) expression (Fig. 1g) in the cells, all of which are major hallmarks of senescence. As expected, S6 (Ser235/236) phosphorylation was elevated in these senescent cells (Fig. 1g). Primary calvarial osteoblasts showed replicative exhaustion at population doubling level (PDL) 25 (Fig. S2a-e). Greater S6 (Ser235/236) phosphorylation was also observed in these replicative senescent cells than in proliferative cells at PDL 3 (Fig. S2e), indicating that mTORC1 is activated during preosteoblast senescence.

mTORC1 activation accelerates senescence in preosteoblasts We next investigated the role of mTORC1 activation in preosteoblast senescence by using mice with osteoblast-specific knockout of $T s c 1$, a negative regulator of mTORC1 ( $\triangle T s c 1$ mice). The $\Delta T s c 1$ mice were fed doxycycline to suppress Osx-Cre expression until they were 12 months old and were sacrificed at the age of 18 months; this treatment resulted in stage-specific preosteoblastic mTORC1 activation at an old age (12-18 months) (Fig. 2a-c). In contrast to the increased bone mass previously observed in young $\Delta T s c 1$ mice, ${ }^{16}$ more serious bone loss was observed in these $\Delta T s c 1$ mice at an old age than in the control Tsc1 flox mice (Fig. 2d, e). The aged $\Delta T s c 1$ mice exhibited decreased serum levels of $\mathrm{N}$-terminal propeptide of type 1 collagen (P1NP) (Fig. S3a) and elevated C-terminal telopeptides of type I collagen (CTX-I) (Fig. S4a), indicating impaired bone formation and increased bone resorption in the $\Delta T s c 1$ mice. Indeed, the numbers of $\mathrm{Osx}^{+}$preosteoblasts (Fig. S3b, c) and mature $\mathrm{Ocn}^{+}$osteoblasts (Fig. S3d, e) were diminished in these old age-specific knockout mice. Double fluorochrome labeling analyses further showed decreased mineral apposition rates (MARs) (Fig. 2f), indicating lower bone formation of osteoblasts in the $\Delta T s c 1$ mice. The number of osteoclasts, which we have reported to be diminished in young $\Delta T s c 1$ mice, ${ }^{16}$ was instead elevated in these old $\Delta T s c 1$ mice (Fig. S4b, c). Immunostaining further revealed elevated p16 expression (Fig. $2 \mathrm{~g}$ ) and proliferative arrest (Fig. $2 \mathrm{~h}$ ) in preosteoblasts of the $\Delta T s \mathrm{~T} 1$ mice. These results suggested that preosteoblastic mTORC1 activation aggravates agerelated bone loss, possibly by accelerating preosteoblast senescence, thus preventing their differentiation into functional osteoblasts and stimulating osteoclast formation.

To further validate these results in vitro, we isolated primary osteoblasts from calvaria and long bones in the $\triangle T s C 1$ mice and induced cell senescence with ROS. As expected, ROS-induced increased expression of the senescence marker p16 (Fig. S5a, d), proliferative arrest (Fig. 2i, Fig. S5e), and elevated SA- $\beta$-Gal activity (Fig. 2j, Fig. 5f) and expression of SASP components (IL-6 and Cxcl1) (Fig. 5b) in osteoblasts. Moreover, ROS-induced senescence impaired the differentiation potential of osteoblasts (Fig. S5c and Fig. 2k). All these hallmarks of osteoblast senescence were exacerbated by mTORC1 activation in the $\triangle T s C 1$ osteoblasts (Fig. 2i-k, Fig. S5), thereby suggesting that mTORC1 activation accelerates senescence in preosteoblasts. We further found that mTORC1 activation exerted consistent inhibitory effects on apoptosis in replicative and senescent preosteoblasts (Fig. S6a), which could not explain the aggravated bone loss in the $\Delta T s c 1$ mice. Together, these results suggested that mTORC1 aggravates agerelated bone loss by accelerating preosteoblast senescence.

mTORC1 inhibition enables preosteoblast escape from senescence

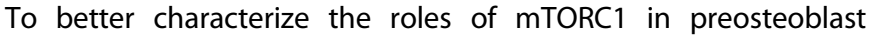
senescence, we established a mouse model with specific mTORC1 inhibition in old age (Fig. 3a) by using the same doxycycline feeding and retreatment procedure as that used in the $\Delta T s c 1$ mice. Micro-CT analysis revealed increased bone volume in the $\Delta$ Raptor mice with mTORC1 inactivation in preosteoblasts (Fig. 3b, c). The stimulated bone formation in the $\Delta$ Raptor mice might have been caused by the alleviated senescence of preosteoblasts (Fig. 3d, e), increased numbers (Fig. 3f, g) and activity (Fig. 3h, i) of osteoblastic lineage cells, and decreased numbers and activity of osteoclasts (Fig. S4).

Consistent with the in vivo results, the $\Delta$ Raptor preosteoblasts exhibited a slower increase in p16 expression (Fig. 4a, Fig. S5d), less pronounced impairment of the proliferative capacity (Fig. 4b, C, Fig. S5e), lower SA- $\beta$-Gal activity (Fig. 4d, e, Fig. S5f), less SASP component secretion (Fig. 4f) and greater differentiation (Fig. 4g) and mineralization (Fig. 4h) when subjected to ROS-induced senescence. Moreover, the $\Delta$ Raptor preosteoblasts showed consistently elevated apoptosis rates in both the replicative and senescent states (Fig. S6b); this finding could not explain the alleviated bone loss in the aged $\Delta$ Raptor mice. On the basis of these results, we concluded that $\mathrm{mTORC} 1$ inhibition alleviated age-related bone loss by enabling preosteoblast escape from senescence.

\section{Plasma membrane depolarization induces preosteoblast} senescence

We then explored the mechanisms responsible for the regulation of preosteoblast senescence by mTORC1. Recently, membrane depolarization has been reported to be associated with mTORC1 and cell senescence. ${ }^{19,20}$ Therefore, we investigated whether potential changes in the plasma membrane might have a role in mTORC1-regulated preosteoblast senescence. To measure their relative plasma membrane potential, we incubated senescent preosteoblasts with the fluorescent dye DiBAC4, which is increasingly taken up by cells with plasma membrane depolarization (and decreases in hyperpolarized cells). The $\Delta T s c 1$ preosteoblasts, which presented accelerated senescence, as described above, showed an elevated DiBAC4 fluorescence intensity, whereas the $\Delta$ Raptor preosteoblasts displayed decreased plasma membrane depolarization (Fig. 5a, b). The patch-clamp experiments revealed the same potential changes in the cells (Fig. $5 \mathrm{c}$ ). 
a

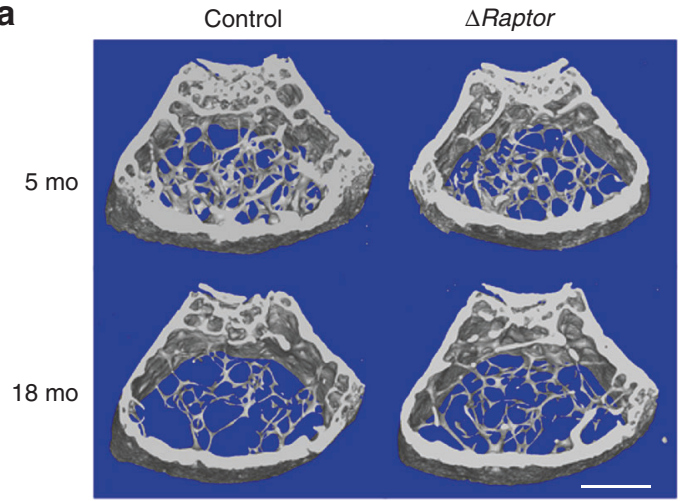

C

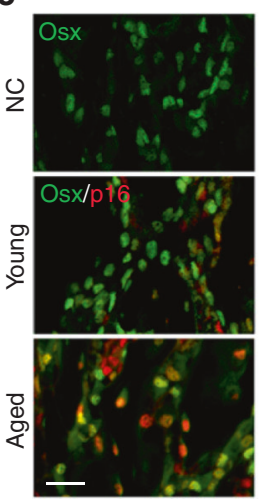

e

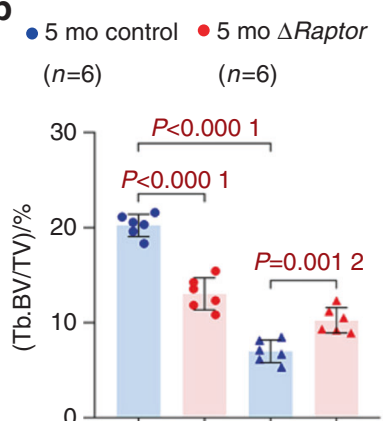

$(n=6)$

$(n=6)$
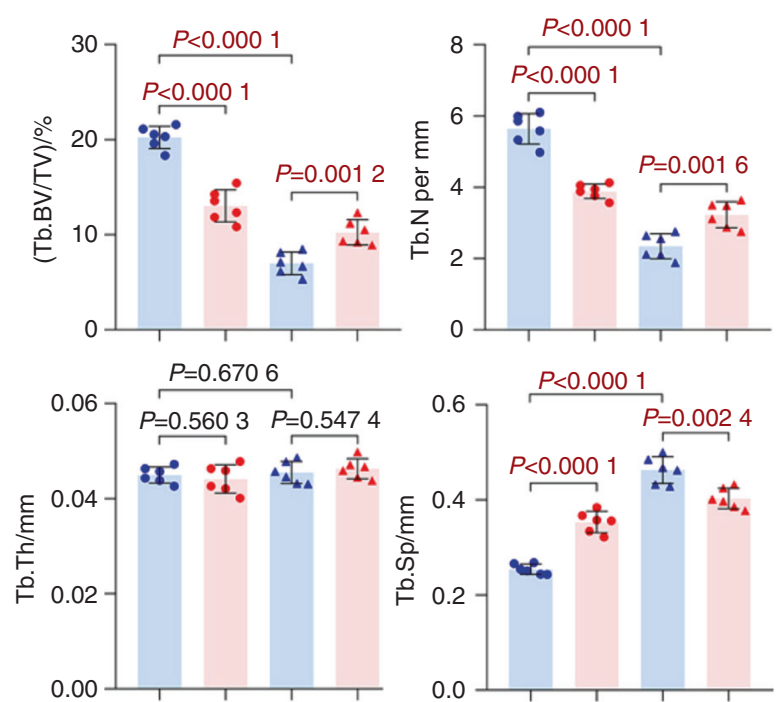
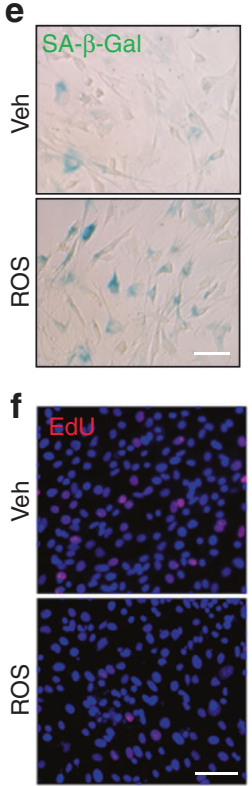
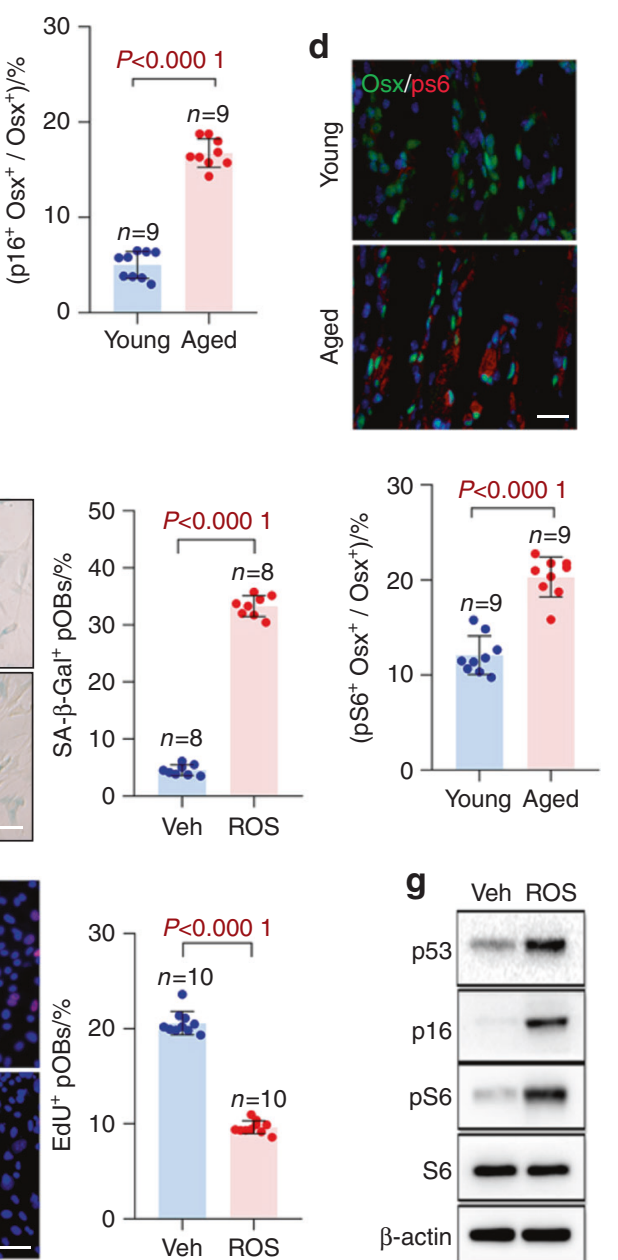

Fig. 1 Senescent preosteoblasts present elevated mTORC1 activity. a Representative microcomputed tomography ( $\mu$ CT) images of femurs of 5-month-old (mo) and 18-month-old (mo) $\Delta$ Raptor mice compared with those of the littermate controls. Scale bar: $500 \mu \mathrm{m}$. b Quantification of trabecular bone volume per total volume (Tb.BV/TV), trabecular number (Tb.N), trabecular separation (Tb.Sp) and trabecular thickness (Tb.Th). Representative images of double immunostaining of Osx plus p16 (c) and Osx plus pS6 (Ser235/236) (d) in the bones of the mice in a. Single Osx staining served as a negative control (NC) for double staining of Osx plus p16 in c. Scale bars: $100 \mu$ m. e The murine preosteoblast cell line MC3T3-E1 was exposed to senescence induction by reactive oxygen species (ROS) or treated with vehicle (Veh) and stained for the expression of senescence-associated $\beta$-galactosidase (SA- $\beta$-gal) 3 days later. Scale bar: $50 \mu \mathrm{m}$. Quantification of the proportion of SA- $\beta$-gal ${ }^{+}$preosteoblasts (pOBs) in each population is shown. $\mathbf{f}$ Representative confocal images of immunostaining of EdU (red) in the cells in e and quantitative analysis of EdU ${ }^{+}$pOBs relative to total cells (\%). Scale bar, $100 \mu \mathrm{m}$. g Western blot analysis of senescence marker (p16 and p53) expression and mTORC1 activity [pS6(Ser235/236)] in the cells in e. Data are shown as the mean \pm SD. The numbers of samples $(n)$ are indicated in each figure panel. $P$ values were determined by two-tailed Student's $t$ test for single comparisons

Interestingly, because depolarization was prevented by pinacidil (Fig. $5 c, d$ ), the $\Delta T s c 1$ preosteoblasts exhibited decreased senescence, as indicated by an increased proliferation rate (Fig. S7a), decreased SA-b-Gal activity (Fig. 5e) and mRNA levels of SASP components (Fig. S7b). We further treated the senescent $\triangle$ Raptor preosteoblasts with $\mathrm{KCl}$, a well-established plasma membrane depolarizer. Forced depolarization in the $\Delta$ Raptor preosteoblasts (Fig. 5c, f) abrogated the beneficial effect of mTORC1 inactivation on cell senescence (Fig. 5g, h and Fig. S8). These results suggested that mTORC1 regulates preosteoblast senescence by controlling plasma membrane potential.

The sodium channel Scn1a mediates plasma membrane depolarization in senescent preosteoblasts

We next investigated the mechanisms underlying the regulation of plasma membrane potential by mTORC1. Ion flow through ion channels causes changes in membrane potential. We were particularly interested in voltage-sensitive sodium $\left(\mathrm{Na}_{\mathrm{v}}\right)$ channels because they mediate fast membrane depolarization ${ }^{23}$ and have been reported to be associated with cell senescence. ${ }^{20}$ To screen for the $\mathrm{Na}_{\mathrm{v}}(\mathrm{s})$ regulated by mTORC1, we analyzed our previous global mRNA expression profiles of the $\triangle T s C 1$ and control calvarial osteoblasts (GSE74781). ${ }^{17}$ Scn1a (sodium channel protein type 1 subunit alpha) showed the most significantly increased expression in the $\Delta T s c 1$ osteoblasts among the $\mathrm{Na}_{\mathrm{v}}(\mathrm{s})$ (Fig. 6a); this result was then verified by quantitative PCR (qPCR) analysis (Fig. S9a). In contrast, Scn1a mRNA levels were lower in the $\triangle$ Raptor osteoblasts (Fig. S9a). The protein levels of Scn1a were also elevated in the $\Delta$ TsC1 but diminished in the $\Delta$ Raptor osteoblasts (Fig. S9b, c), thus indicating positive regulation of Scn1a by mTORC1.

How does mTORC1 regulate Scn 1 a expression? The transcription factor C/EBPa is known to be positively regulated by $\mathrm{mTORC} 1^{24}$ and 
a

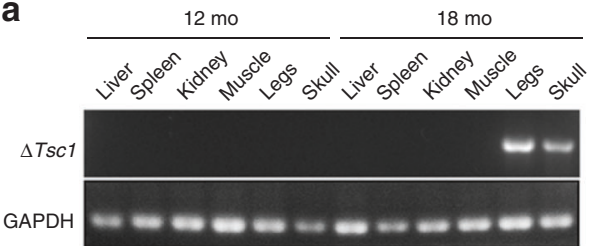

e

$$
\begin{array}{lc}
\begin{array}{c}
\text { - } 12 \text { mo control } \\
(n=6)
\end{array} & \begin{array}{c}
12 \mathrm{mo} \Delta T s c 1 \\
(n=6)
\end{array} \\
\begin{array}{l}
12 \mathrm{mo} \text { control } \\
(n=6)
\end{array} & \begin{array}{c}
12 \mathrm{mo} \Delta T s c 1 \\
(n=6)
\end{array}
\end{array}
$$
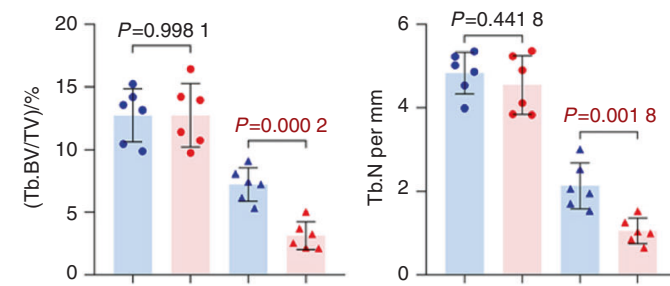

b

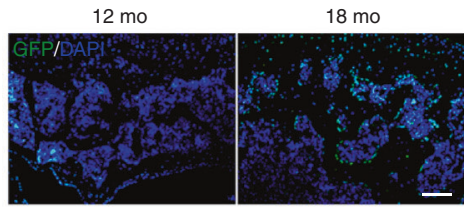

d Control

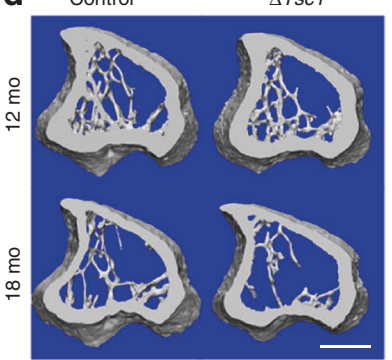

C
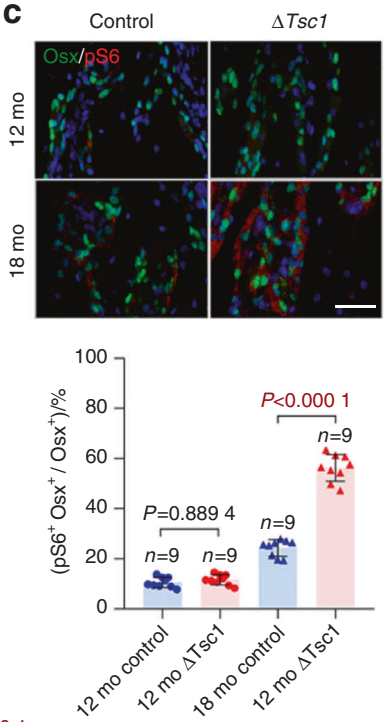

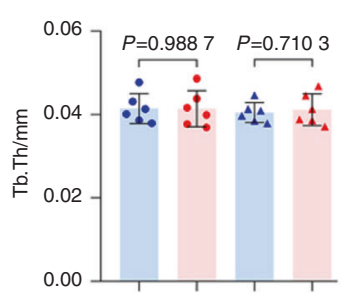

f
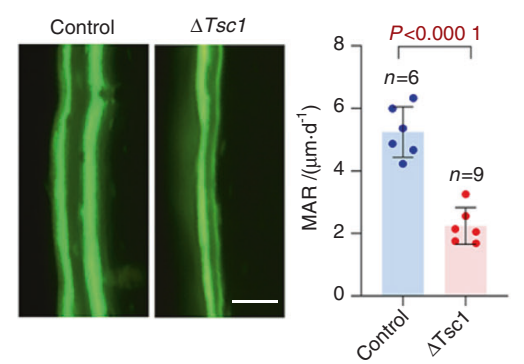

g

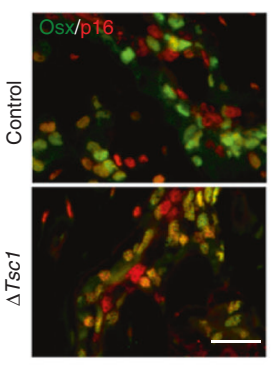

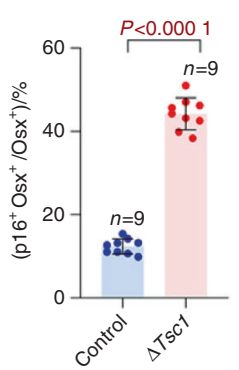

i

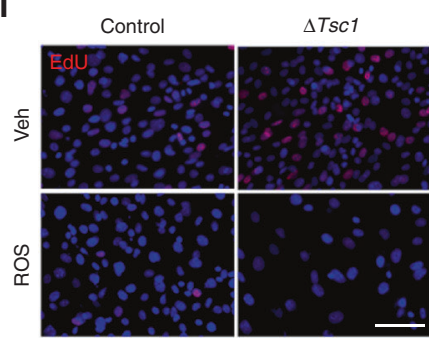

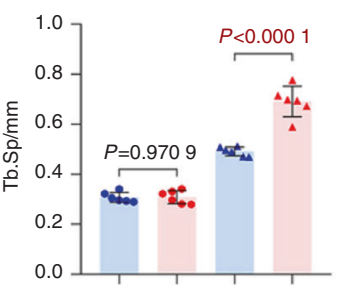

k
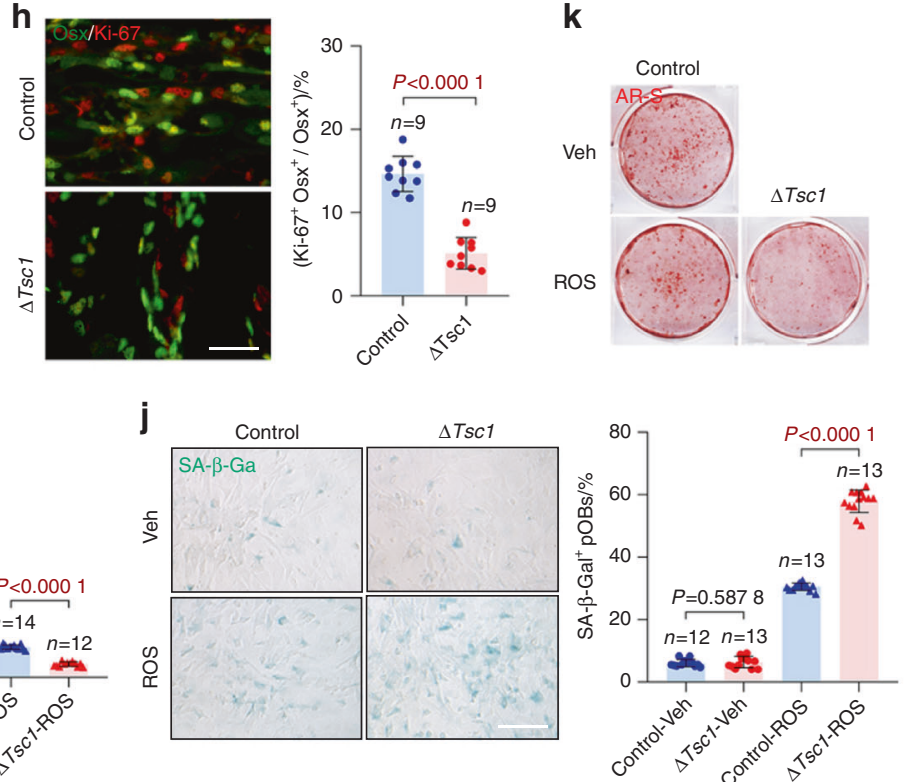

Fig. 2 Hyperactive mTORC1 aggravates preosteoblast senescence and age-related bone loss. a PCR analysis of Tsc1 allele recombination in tissues from 12- and 18-month-old $\triangle T S C 1$ mice. Primers for GAPDH were used as a loading control. b Immunostaining of GFP, which indicated Osx-Cre expression, in bone sections of the mice in a. Scale bar, $200 \mu \mathrm{m}$. c Double immunostaining of Osx and pS6 and quantification of $\mathrm{pS}^{+}$preosteoblasts $\left(\mathrm{pS}^{+} \mathrm{Osx}{ }^{+}\right.$) relative to total preosteoblasts $\left(\mathrm{Osx}^{+}\right)$in bone sections of the mice in a. Scale bar, $100 \mu \mathrm{m}$. d Representative microcomputed tomography $(\mu \mathrm{CT})$ images of 12- and 18-month-old $\Delta T s c 1$ tibias compared with those of the littermate controls, Scale bar, $500 \mu \mathrm{m}$. e Quantification of Tb. BV/TV, Tb.N, Tb.Sp, and Tb.Th. f Representative images of calcein labels and quantification of the mineral apposition rate (MAR) in femurs from the 18-month-old $\Delta T s c 1$ mice. Scale bar $=50 \mu \mathrm{m}$. Double immunostaining of Osx plus p16 (g) and Osx plus Ki-67 (h) in tibias of the 18-month-old $\Delta T s c 1$ mice. Double positively stained cells were quantified. Scale bar: $100 \mu \mathrm{m}$. In primary calvarial osteoblasts isolated from neonatal $\Delta T s c 1$ and $T s c 1^{\text {fl/f }}$ mice, senescence was induced by ROS. After 3 days, (i) the cells were immunostained for EdU and analyzed for EdU ${ }^{+}$cells relative to total cells. Scale bar, $50 \mu$ m. j Representative images of SA- $\beta$-gal staining and quantification of the proportion of SA- $\beta$-gal-positive cells in the senescence-induced cells. Scale bar, $100 \mu \mathrm{m}$. k After induction of osteogenic differentiation, cells were subjected to AR-S staining on Day 14 after differentiation induction. Data are shown as the mean \pm SD. The numbers of samples $(n)$ are indicated in each figure panel. $P$ values were determined with two-tailed Student's $t$ test for single comparisons 
a
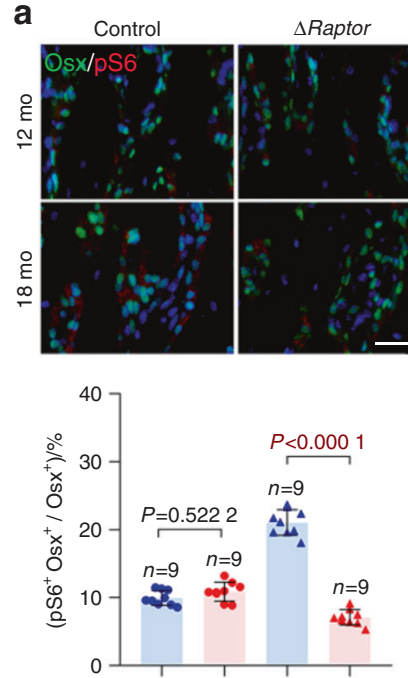

b
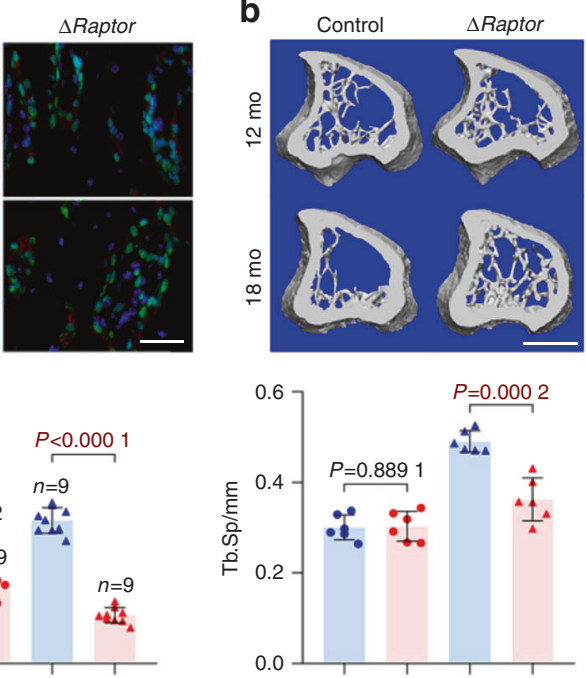

C
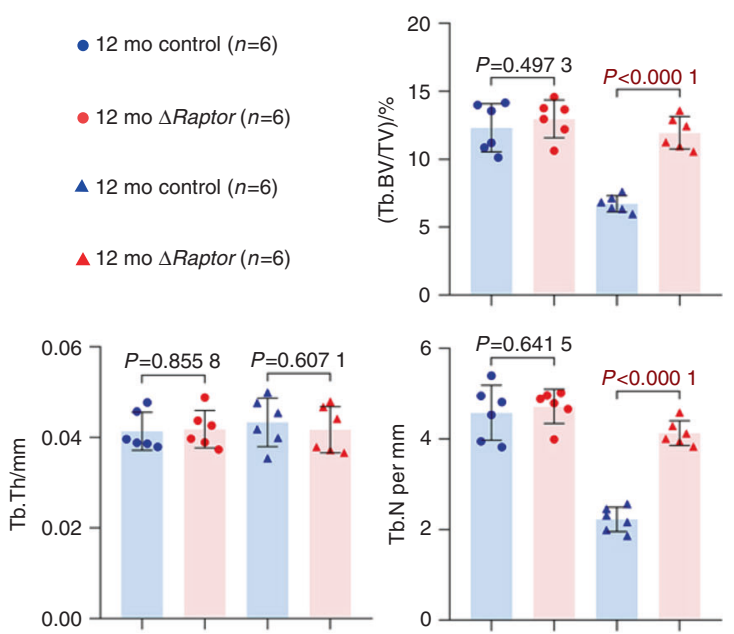

d
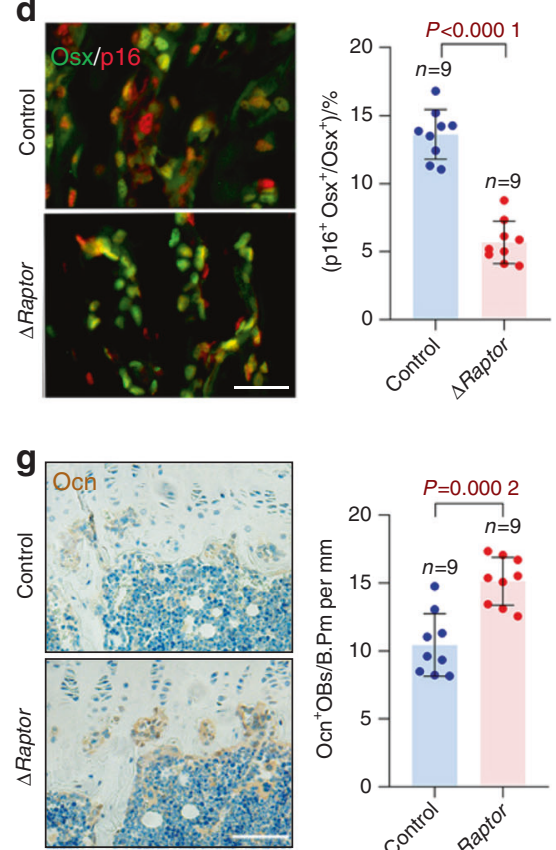

e
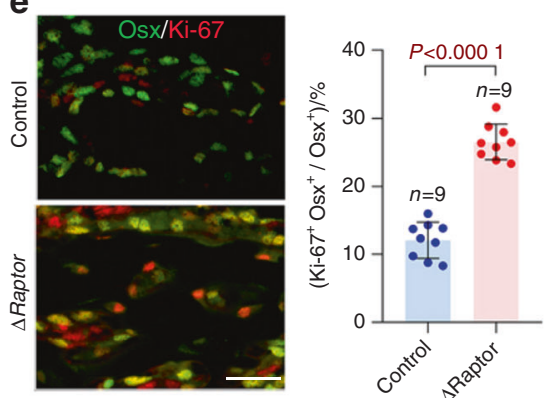

h

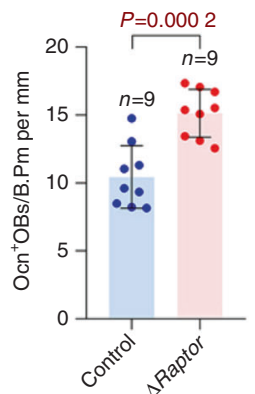

f
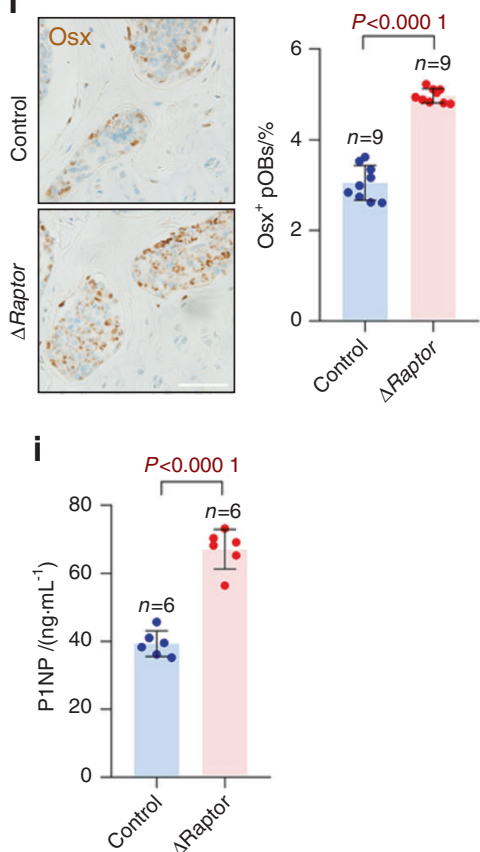

Fig. 3 mTORC1 inactivation alleviates preosteoblast senescence and bone loss. a Double immunostaining of Osx and pS6 and quantification of $\mathrm{pS}^{+}$preosteoblasts $\left(\mathrm{pS6}^{+} \mathrm{Osx}^{+}\right)$relative to total preosteoblasts $\left(\mathrm{Osx}{ }^{+}\right)$in the tibias of 12 - and 18-month-old $\Delta$ Raptor mice. Scale bar, $100 \mu \mathrm{m}$. Representative microcomputed tomography $(\mu \mathrm{CT})$ images (b) and quantification of trabecular bone (c) in the 12- and 18-month-old $\Delta$ Raptor tibias compared with those from the littermate controls. Scale bar, $500 \mu \mathrm{m}$. Double immunostaining of Osx plus p16 (d) and Osx plus $\mathrm{Ki}-67$ (e) in tibias. Double positively stained cells were quantified. Scale bars, $100 \mu \mathrm{m}$. IHC staining for Osx (f) and osteocalcin (Ocn) (g) in the tibias of the 18-month-old $\Delta$ Raptor mice and their littermate controls. Scale bar: $100 \mu \mathrm{m}$. Osx-positive preosteoblasts (Osx ${ }^{+}$pOBs) were quantified as cell numbers per total cells in the bone marrow. Ocn-positive mature osteoblasts (Ocn ${ }^{+} \mathrm{OBs}$ ) on the bone surface were measured as cells per millimeter of perimeter in the sections (/B.Pm). $\mathbf{h}$ Representative images of calcein labels and quantification of the mineral apposition rate (MAR) in femurs from the 18-month-old $\Delta$ Raptor mice and their littermate controls. Scale bar $=50 \mu \mathrm{m}$. i Serum levels of P1NP in the 18-month-old $\Delta$ Raptor mice and their littermate controls determined by ELISAs. Data are shown as the mean \pm SD. The numbers of samples $(n)$ are indicated in each figure panel. $P$ values were determined by two-tailed Student's $t$ test for single comparisons

has been shown to bind $5^{\prime}$-untranslated exons (UE) of SCN1A and substantially enhance its transcription. ${ }^{25}$ We therefore investigated whether C/EBPa mediates the regulation of Scn1a by mTORC1. Indeed, we found that both the mRNA (Fig. S9d) and protein (Fig. S9e) levels of C/EBPa were elevated in the $\Delta T s C 1$ but diminished in the $\Delta$ Raptor osteoblasts. By using transcription factor databases, we predicted the presence of a putative C/EBPa binding site on the $5^{\prime}$-UE of Scn1a (Fig. 6b). In ChIP assays, C/EBPa was further demonstrated to bind the $5^{\prime}$-UE of Scn1a in mouse preosteoblasts (Fig. $6 \mathrm{c}$ ). mTORC1 activation promoted and mTORC1 inhibition attenuated this binding (Fig. 6c). Importantly, the increased expression of Scn1a was reversed by siRNA downregulation of $\mathrm{C} / \mathrm{EBPa}$ expression in the $\Delta T s C 1$ preosteoblasts (Fig. $\mathrm{S} 9 \mathrm{f})$, thus confirming that $\mathrm{C} / \mathrm{EBPa}$ is involved in the regulation of Scn1a gene transcription by mTORC1.

We then determined whether Scn1a mediates the regulation of membrane potential and cell senescence by mTORC1. Knockdown of Scn1a expression by siRNA (Fig. 6d) alleviated membrane depolarization (Fig. 6e) and reversed the senescent phenotypes (Fig. $6 \mathrm{f}-\mathrm{h}$ ) in the $\Delta T s c 1$ preosteoblasts. To validate these results in vivo, we 


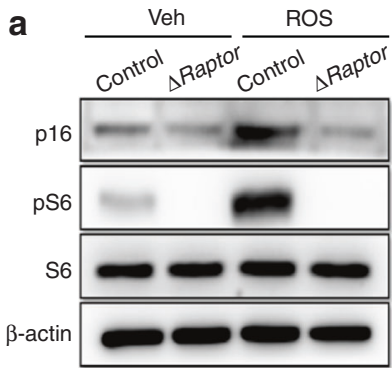

b
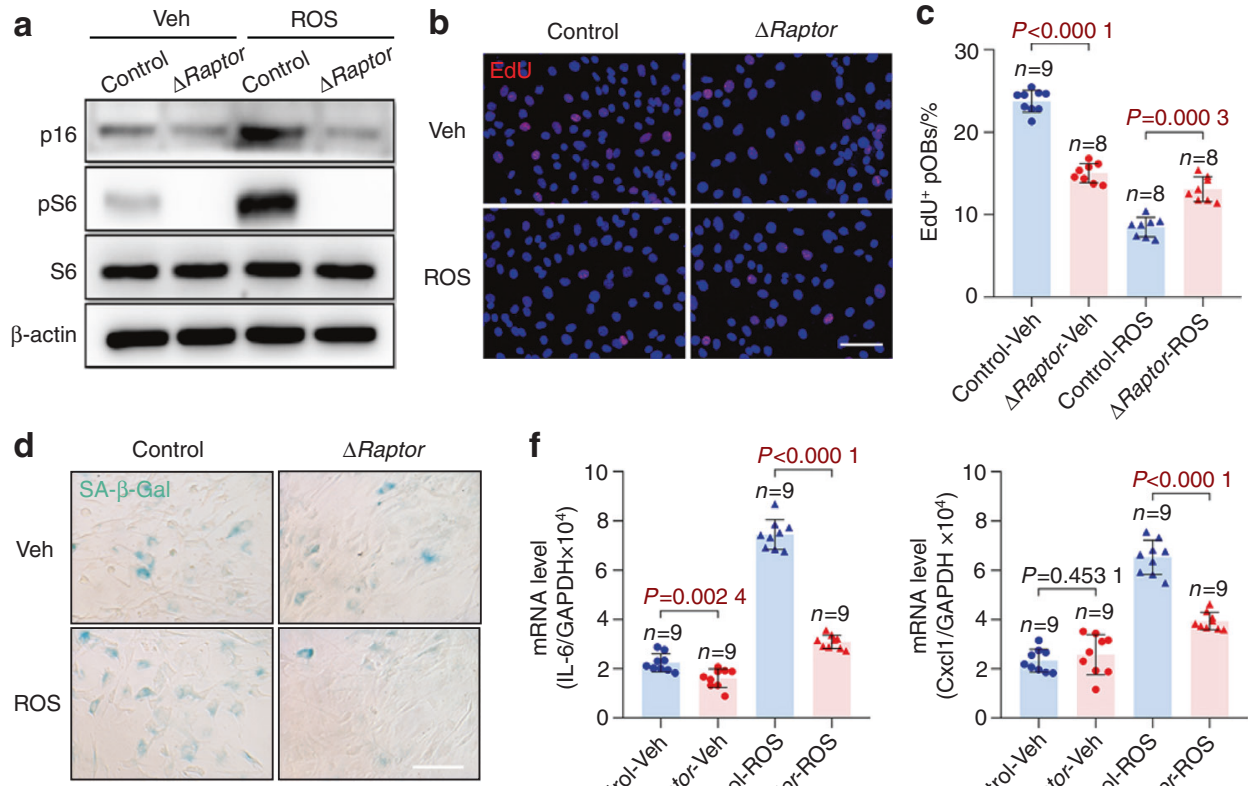

$\mathbf{f}$

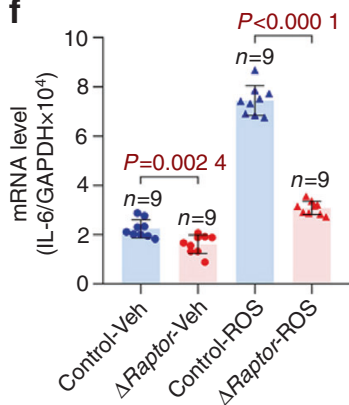

e

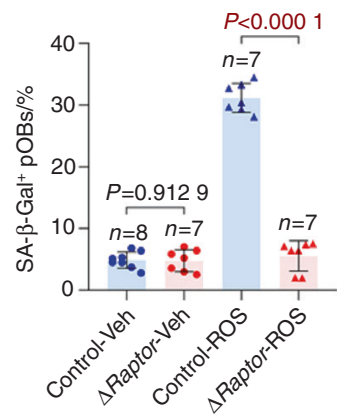

g

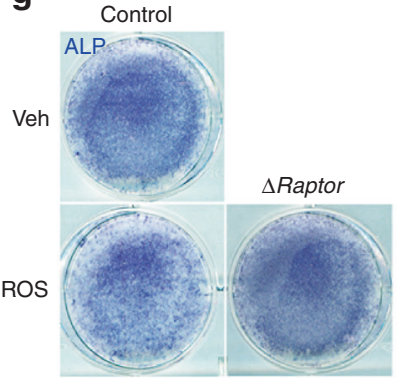

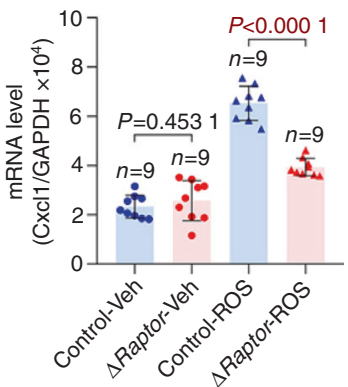

h

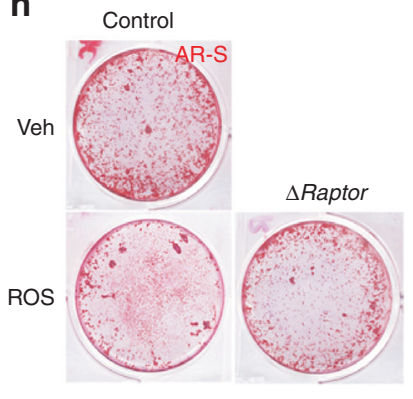

Fig. 4 mTORC1 inhibition enables preosteoblast escape from senescence in vitro. a Primary calvarial osteoblasts isolated from neonatal $\Delta$ Raptor and Raptor ${ }^{\mathrm{fl} / \mathrm{fl}}$ mice were induced to undergo senescence by ROS. After 3 days, the cells were analyzed for senescence marker (p16 and p53) expression and mTORC1 activity (pS6) with western blotting. b Immunostaining of EdU in the senescence-induced cells in a. Scale bar, $50 \mu \mathrm{m}$. c Quantitative analysis of EdU ${ }^{+}$cells relative to total cells. d Representative images of SA- $\beta$-gal staining of the senescence-induced cells in a. Scale bar, $100 \mu \mathrm{m}$. e Quantification of the proportion of SA- $\beta$-gal-positive cells in each population. $\mathbf{f}$ qPCR analysis of IL-6 and Cxcl 1 mRNA in the primary preosteoblasts in a. The cells in a were then induced to undergo osteogenic differentiation and subjected to (g) ALP staining or (h) AR-S staining on Day 7 or 14, respectively, after induction of differentiation. Data are shown as the mean \pm SD. The numbers of samples $(n)$ are indicated in each figure panel. $P$ values were determined with two-tailed Student's $t$ test for single comparisons

injected the aged $\Delta T s c 1$ mice with adenovirus encoding si-Scn1a. With interference with Scn1a expression in preosteoblasts (Fig. 6i), the $\Delta T s c 1$ mice exhibited alleviated preosteoblast senescence (Fig. 6j) and increased bone mass (Fig. 6k, l). Histomorphometric measurements further showed increased mineral apposition rates (MARs) indicative of higher bone formation activity of osteoblasts in the $\Delta T s c 1$ mice receiving si-Scn1a (Fig. $6 \mathrm{~m}$ ). These results demonstrated that Scn1a functionally mediated plasma membrane potential changes and cellular senescence in preosteoblasts.

Prosenescence stress activates Scn1a by inhibiting its phosphorylation by PKA

Interestingly, we further found that the regulation of membrane depolarization by mTORC1 was confined to senescent osteoblasts, because mTORC1 activation or inhibition did not cause a significant change in membrane potential in replicative osteoblasts without senescence induction (Fig. 7a). This result suggested that the permeability of Scn 1 a to $\mathrm{Na}^{+}$might be controlled by prosenescence stress. The function of Scn1a in mediating sodium currents is suppressed by its phosphorylation by the protein kinase PKA. ${ }^{26-28}$ Coincidentally, PKA activity has been reported to be diminished in senescent cells. ${ }^{29}$ Therefore, we reasoned that prosenescent stress might activate Scn1a by inhibiting its phosphorylation through suppression of PKA. As expected, PKA activity was found to decrease in senescent preosteoblasts (Fig. 7b). PKA activation by F/I (forskolin + IBMx) (Fig. 7b) abolished the membrane depolarization (Fig. 7c) and senescent phenotype (Fig. 7d and Fig. S10) of preosteoblasts induced by ROS. In contrast, the PKA inhibitor $\mathrm{H}-89$ alone was sufficient to induce membrane depolarization (Fig. 7e, f) and senescence (Fig. 7g, h and Fig. S11) in preosteoblasts. Moreover, the $\Delta T s c 1$ preosteoblasts exhibited greater depolarization (Fig. 7f) and more significant senescent phenotypes (Fig. $7 g$, $\mathrm{h}$ and Fig. S11) than the wild-type preosteoblasts after treatment with $\mathrm{H}-89$. These results verified that prosenescent stress activates Scn1a by inhibiting its phosphorylation through suppression of PKA, thereby explaining the unique state of membrane depolarization in senescence and the age-specific effects of mTORC1 on osteoblasts in old mice.

Plasma membrane depolarization increases $\mathrm{Ca}^{2+}$ influx and activates NFAT/ATF3/p53 signaling, thereby inducing preosteoblast senescence

Finally, we investigated the mechanisms through which plasma membrane depolarization contributes to preosteoblast senescence. Plasma membrane depolarization is known to activate 

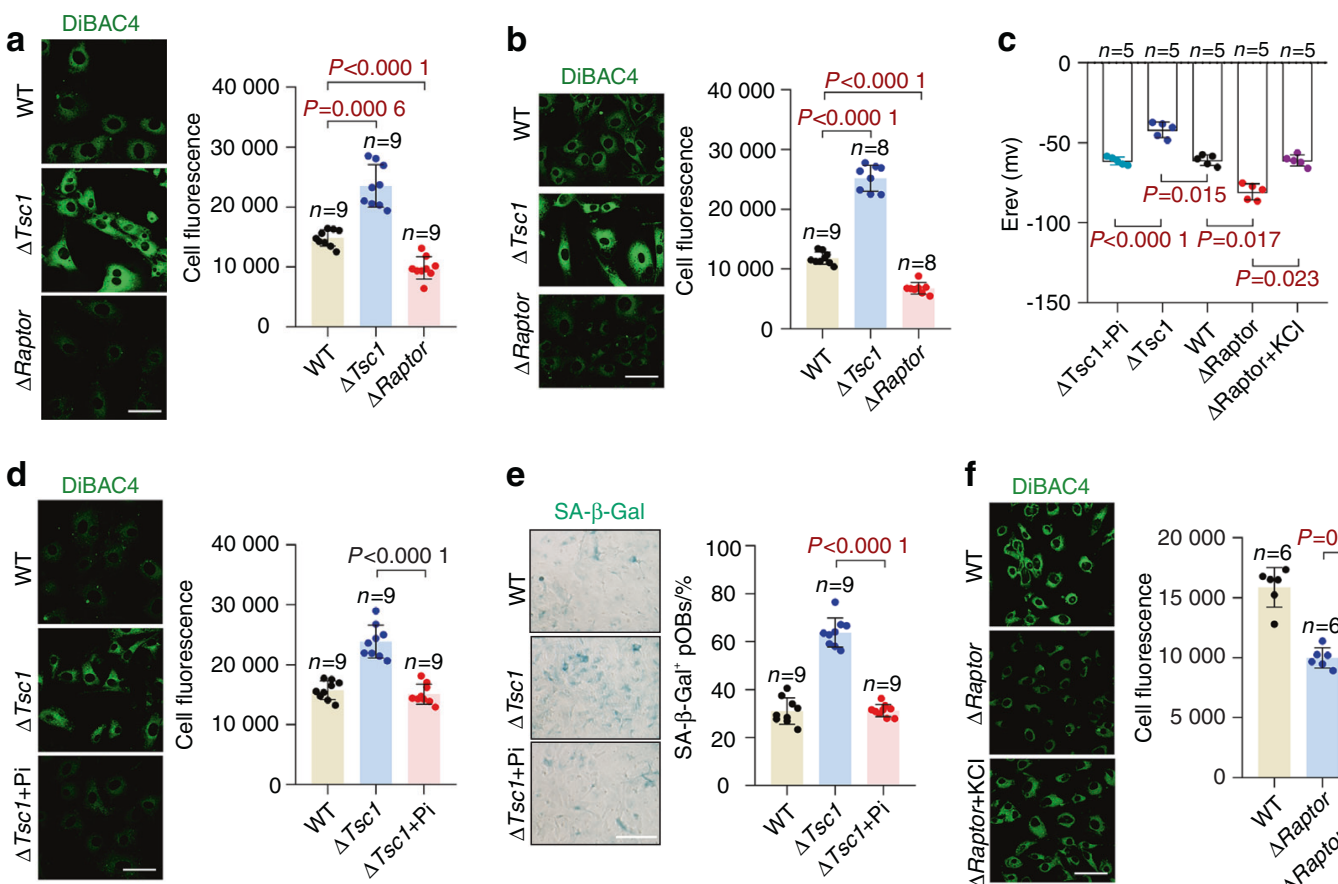

e
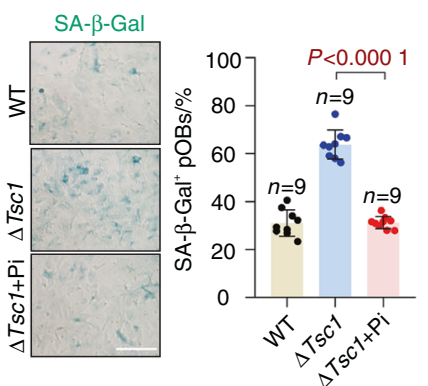

f $\quad$ DiBAC4
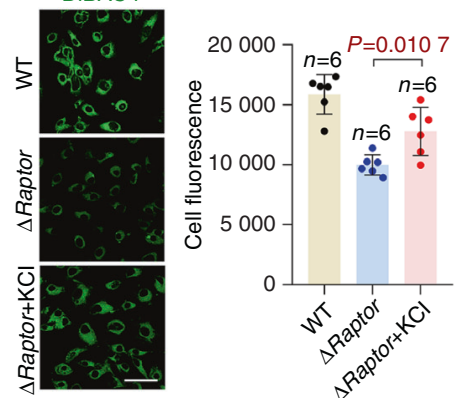

g EdU

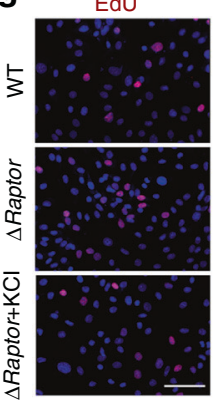

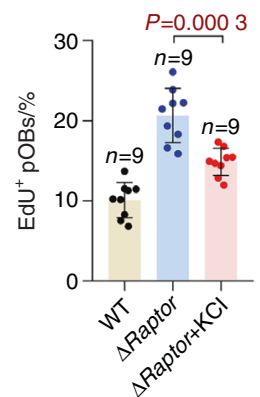
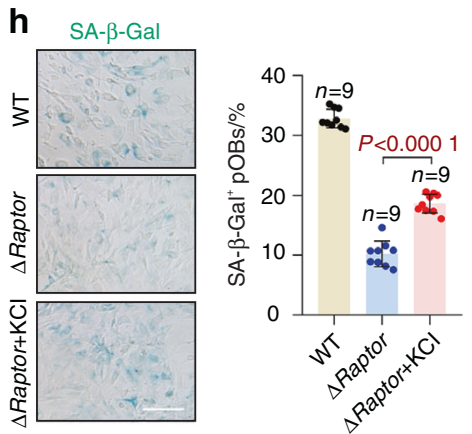

Fig. 5 Plasma membrane depolarization contributes to preosteoblast senescence. Senescent wild-type (WT), $\Delta T s c 1$, and $\Delta$ Raptor preosteoblasts isolated from calvaria (a) or long bones (b) were incubated with fluorescent DiBAC4 dye and photographed under a confocal microscope. Scale bars, $50 \mu \mathrm{m}$. Cell fluorescence was measured as the mean corrected total cell fluorescence (CTCF) of all the cells in nine different images taken at $\times 400$ magnification in ImageJ. Depolarization is indicated by increased fluorescence. $\mathbf{c}$ Histograms showing the mean reversal potentials $\left(E_{\text {rev }}\right)$ of ramp membrane currents determined by the patch-clamp technique in the different indicated preosteoblasts. d Representative confocal images of DiBAC4 fluorescence in the senescent $\Delta T s c 1$ preosteoblasts treated with pinacidil (Pi). Scale bar, $50 \mu \mathrm{m}$. The fluorescence intensity of the cells was measured. e SA- $\beta$-gal staining of the cells in $\mathbf{d}$ and quantification of the proportion of SA- $\beta$-gal-positive cells in each population. Scale bar, $100 \mu \mathrm{m}$. f DiBAC4 fluorescence in the senescent $\Delta$ Raptor preosteoblasts treated with $\mathrm{KCl}$. Relative plasma membrane potentials were measured. $\mathbf{g}$ Immunostaining of EdU in the cells in $\mathbf{f}$ and quantitative analysis of EdU ${ }^{+}$cells relative to total cells. Scale bar, $100 \mu \mathrm{m}$. $\mathbf{h}$ SA- $\beta$-gal staining of the cells in $\mathbf{f}$ and quantification of the proportion of SA- $\beta$-gal-positive cells. Scale bar, $100 \mu \mathrm{m}$. Data are shown as the mean \pm SD. The numbers of samples $(n)$ are indicated in each figure panel. $P$ values were determined by two-tailed Student's $t$ test for single comparisons

voltage-gated calcium channels and increase intracellular calcium, ${ }^{30}$ and this increased calcium can promote cell senescence. ${ }^{31,32}$ Indeed, $\mathrm{Ca}_{\mathrm{v}} 1.2$ was expressed constitutively in preosteoblasts (Fig. 8a). Moreover, the senescent $\Delta T s c 1$ preosteoblasts displayed elevated cytosolic calcium levels, which were then decreased by the repolarization induced by pinacidil. In contrast, calcium levels were diminished in the senescent $\Delta$ Raptor preosteoblasts but were increased by $\mathrm{KCl}$-induced depolarization (Fig. 8b, c). Because $\mathrm{Ca}_{\mathrm{v}} 1.2$ showed consistent expression among the two sets of transgenic preosteoblasts and the wild-type controls (Fig. 8a), we speculated that plasma membrane depolarization might increase $\mathrm{Ca}^{2+}$ influx by activating calcium channels.

Increased cytosolic calcium induces cell senescence by triggering NFAT dephosphorylation and its translocation to the nucleus. ${ }^{33}$ NFATc1 inhibits the expression of ATF3, which in turn suppresses the expression of p53 and other senescence-related markers. ${ }^{34}$ p53 induces transcription of the cyclin-dependent kinase inhibitor p21. In turn, p21 blocks CDK4/6 activity, thus resulting in decreased cyclin $\mathrm{D}$ levels, hypophosphorylated $\mathrm{Rb}$ and cell cycle exit. ${ }^{35}$ Consistent with these findings, we observed that translocation of NFATC1 to the nucleus was promoted in the senescent $\triangle T s c 1$ preosteoblasts (Fig. 8d). Consequently, ATF3 expression was diminished in the $\Delta T s c 1$ preosteoblasts in vitro (Fig. 8e) and in bone (Fig. 8f), thereby further increasing p53 expression and decreasing cyclin $\mathrm{D}$ levels and $\mathrm{Rb}$ phosphorylation in the cells (Fig. 8e). In contrast, the senescent $\Delta$ Raptor cells exhibited inhibited NFAT/ATF3/p53 signaling and increased cyclin $\mathrm{D}$ levels and Rb phosphorylation (Fig. 8d-f). After interference with p53 expression, the $\Delta T s c 1$ preosteoblasts showed increased cyclin $\mathrm{D} 1$ levels and $\mathrm{Rb}$ phosphorylation (Fig. 8g) and alleviated senescent phenotypes (Fig. $8 \mathrm{~h}-\mathrm{j}$ ). Moreover, the addition of a 
b

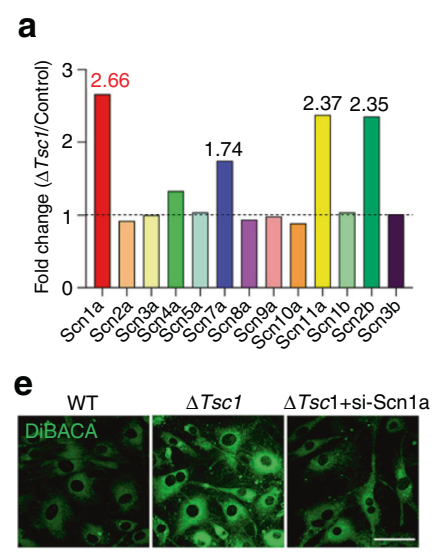

f
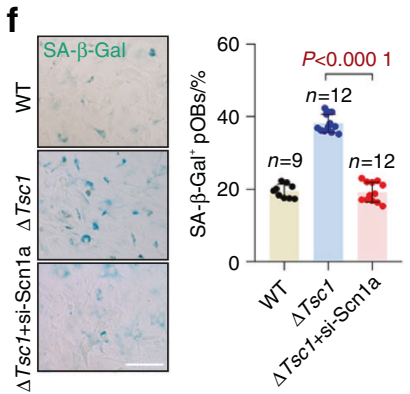
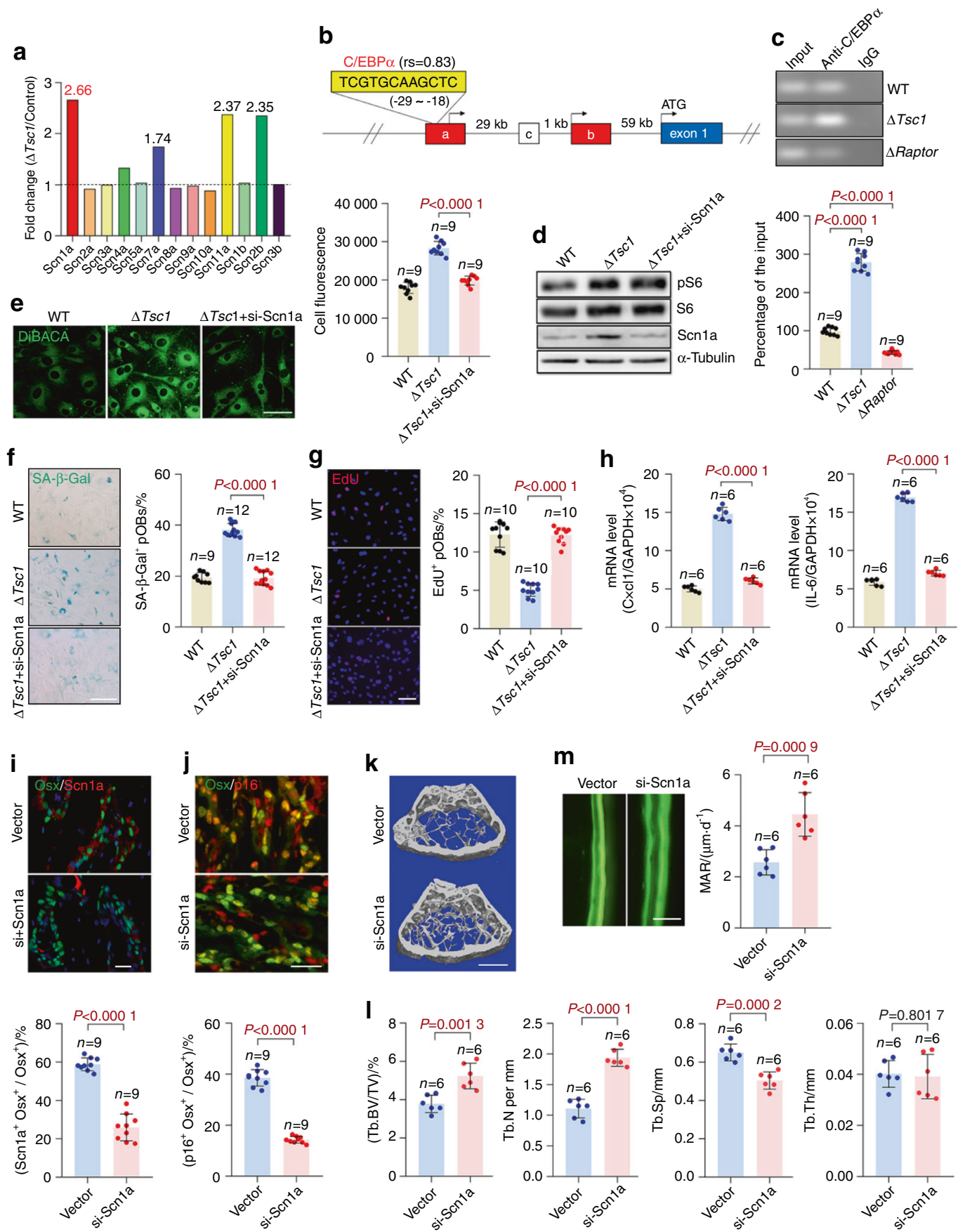

m
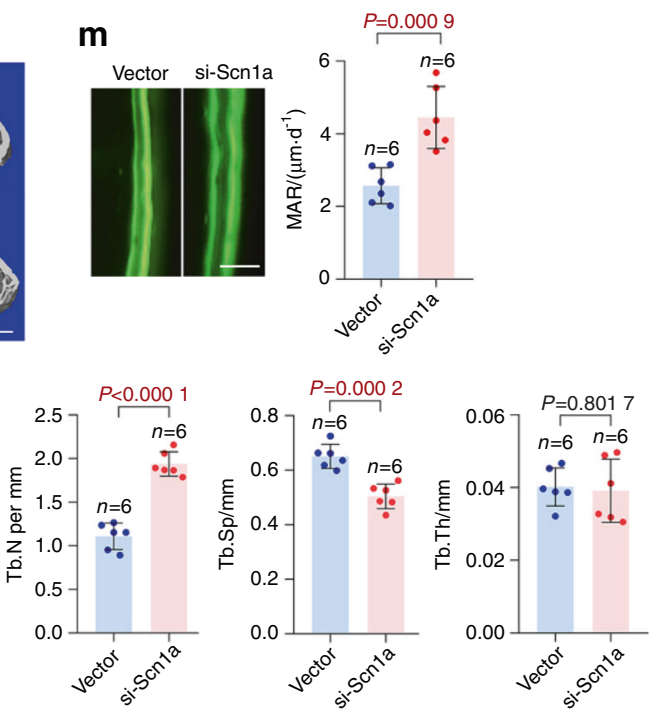

Fig. 6 The sodium channel Scn1a mediates plasma membrane depolarization in senescent preosteoblasts. a mRNA expression of voltage-sensitive sodium channels in the $\triangle T s c 1$ and control calvarial preosteoblasts indicated by our previous global mRNA expression profile (GSE74781). b Binding site of C/EBP $\alpha$ in the 5'-UE of Scn1a (yellow box) predicted by the JASPAR database (http://jaspar.genereg. net). rs: JASPAR relative scores, which are defined as 1 for the maximum-likelihood sequence. Boxes represent exons: blue coding exons, red noncoding exons conserved between humans and mice, white noncoding exons identified in either human or mouse transcripts. Noncoding exons are named alphabetically, and the first coding exon (exon 1) of the Scn1a gene is indicated. Genomic distances between exons are indicated. c Binding of C/EBP $\alpha$ to the $5^{\prime}$-UE sequence of Scn 1 a in vivo, determined by a ChIP assay using the $\Delta T s c 1$ and $\Delta$ Raptor cells and anti-C/EBP $\alpha$ antibody or IgG. The ChIP samples were then subjected to qPCR with the Scn1a $5^{\prime}-\mathrm{UE}$ primers. The percentage of the input of the sample by using the anti-C/EBP $\alpha$ antibody in the wild-type cells was normalized to 100 . The $\Delta T s C 1$ osteoblasts were transfected with Scn1a siRNA and subjected to (d) Scn1a detection with western blotting, (e) measurement of relative plasma membrane potential with DiBAC4 dye, (f) SA- $\beta$-gal staining and quantification of the proportion of SA- $\beta$-gal-positive cells, (g) immunostaining of EdU and quantitative analysis of EdU ${ }^{+}$cells relative to total cells, and (h) qPCR analysis of IL-6 and Cxcl1 mRNA. Double immunostaining of Osx plus Scn $1 \mathrm{a}$ (i) and Osx plus p16 (j) in the tibias of the 18-month-old $\Delta T s c 1$ mice injected with adenovirus encoding si-Scn1a for 1 month. Double positively stained cells were quantified. Representative $\mu C T$ images (k) and quantification of trabecular bone (I) in the mice. (m) Representative images of calcein labels and quantification of the mineral apposition rate (MAR) in femurs from the 18-month-old $\Delta T s c 1$ mice receiving si-Scn 1 a. Scale bars: $50 \mu \mathrm{m}$ in e, $\mathbf{g}, \mathbf{m} ; 100 \mu \mathrm{m}$ in $\mathbf{i}$, j; and $500 \mu \mathrm{m}$ in $\mathbf{k}$. Data are shown as the mean \pm SD. The numbers of samples $(n)$ are indicated in each figure panel. $P$ values were determined with two-tailed Student's $t$ test for single comparisons 


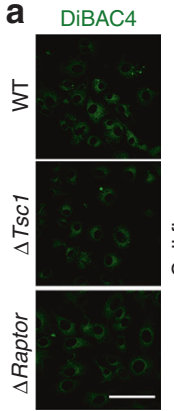

d SA- $\beta-G a l$
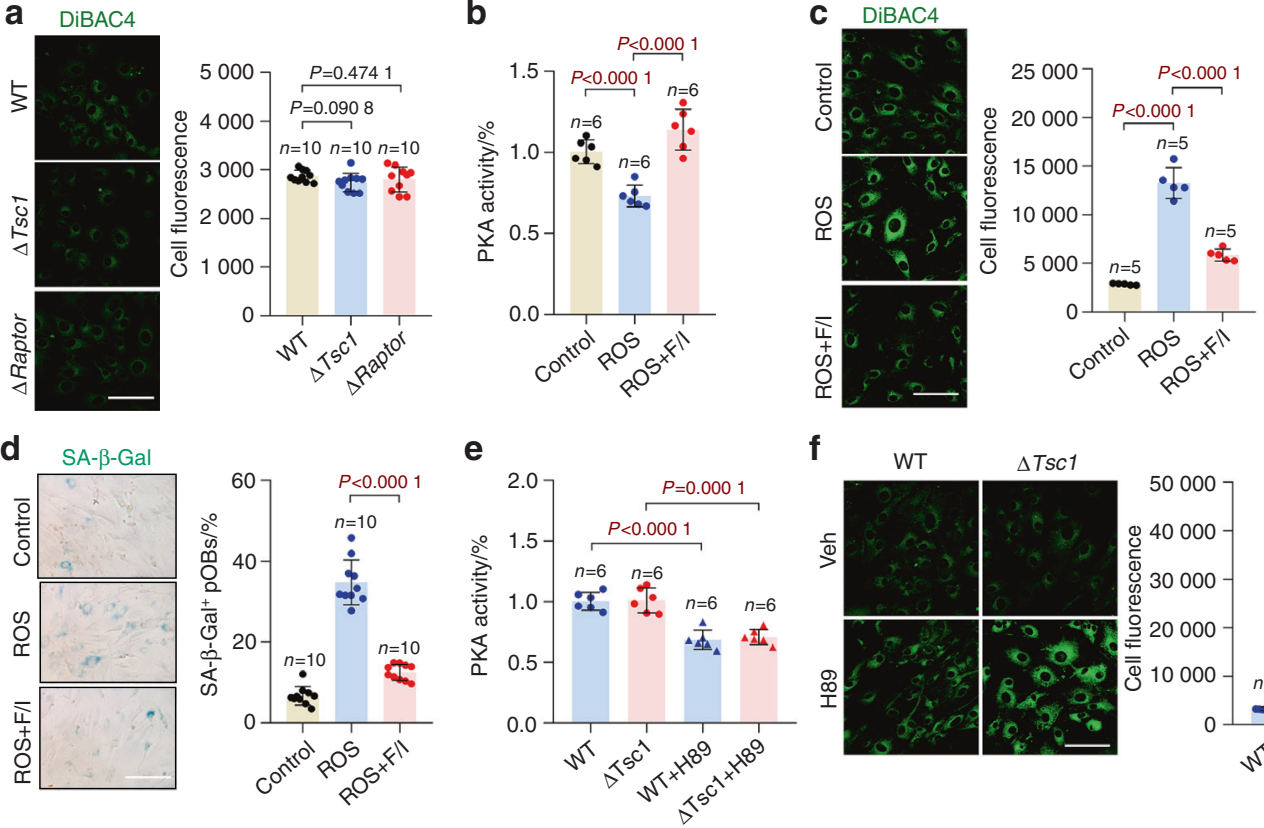

g

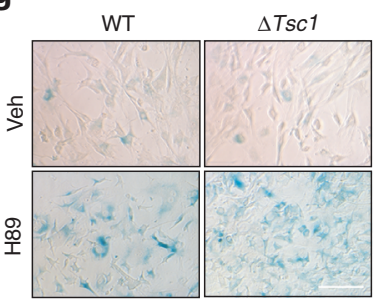

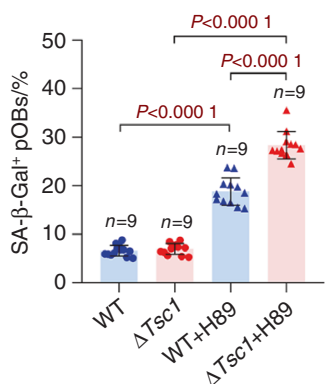

h

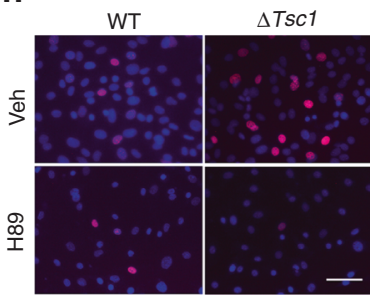

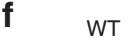
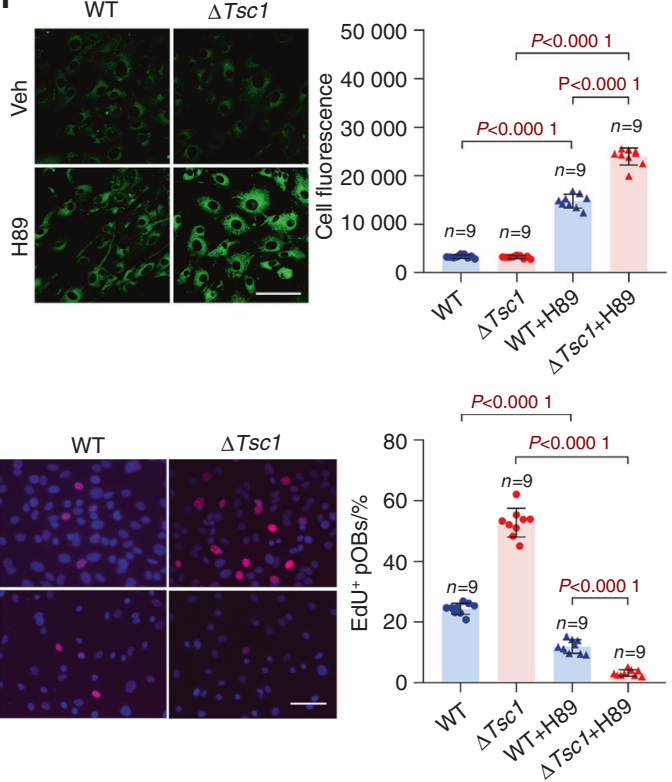

Fig. 7 Prosenescent stress activates Scn 1a by inhibiting PKA. a Replicative wild-type (WT), $\Delta T s c 1$ and $\Delta$ Raptor calvarial osteoblasts were incubated with the fluorescent DiBAC4 dye and photographed under a confocal microscope. Scale bar, $50 \mu \mathrm{m}$. Relative plasma membrane potentials were measured. ROS-induced senescent wild-type preosteoblasts were treated with F/I (forskolin + IBMx, PKA activator) or left untreated. The cells were subjected to (b) measurement of total cellular PKA activities, (c) DiBAC4 staining and measurement of relative plasma membrane potentials. Scale bar, $50 \mu \mathrm{m}$. d SA- $\beta$-gal staining of cells in $\mathrm{b}$ and quantification of the proportion of SA- $\beta$-gal-positive cells in each population. Scale bar, $100 \mu \mathrm{m}$. Replicative WT and $\Delta T s c 1$ osteoblasts were treated with H-89 (PKA inhibitor) and were subjected to (e) measurement of total cellular PKA activities, (f) DiBAC4 staining and measurement of relative plasma membrane potentials. Scale bar, $50 \mu \mathrm{m}$. g SA- $\beta$-gal staining of cells in e and quantification of the proportion of SA- $\beta$-gal-positive cells in each population. Scale bar, $100 \mu \mathrm{m}$. $\mathbf{h}$ Immunostaining of EdU in cells in e and quantitative analysis of EdU ${ }^{+}$cells relative to total cells. Scale bar, $100 \mu \mathrm{m}$. Data are shown as the mean \pm SD. The numbers of samples $(n)$ are indicated in each figure panel. $P$ values were determined by two-tailed Student's $t$ test for single comparisons

calcium chelator (BAPTA-AM) abolished the activation of NFATc1/ ATF3/p53 signaling (Fig. 8k, I) and the increase in senescence markers (Fig. $8 \mathrm{~m}-0$ ) in the $\Delta T s c 1$ preosteoblasts. These results suggested that plasma membrane depolarization causes preosteoblast senescence by increasing $\mathrm{Ca}^{2+}$ influx and activating NFAT/ATF3/p53 signaling.

\section{DISCUSSION}

Understanding the mechanisms responsible for preosteoblast senescence is critical for the development of therapeutic interventions for age-related bone loss. Using mouse models with mTORC1 activation or inhibition in preosteoblasts during old age, we identified the sodium channel Scn1a as a novel regulator of preosteoblast senescence. We found that the plasma membranes in senescent preosteoblasts are depolarized, which promotes cell senescence by increasing $\mathrm{Ca}^{2+}$ influx and activating downstream NFAT/ATF3/p53 signaling. Scn 1 a promotes the senescence of preosteoblasts by driving depolarization of the plasma membrane. Scn1a expression is positively regulated by mTORC1 upstream of C/EBPa, whereas its permeability to $\mathrm{Na}^{+}$is tightly regulated by
PKA activity during cell senescence. Prosenescent stresses elevate the permeability of $\mathrm{Scn} 1 \mathrm{a}$ to $\mathrm{Na}^{+}$by suppressing PKA activity and induce depolarization specifically in senescent preosteoblasts (Fig. 9). Thus, Scn1a is a novel regulator of preosteoblast senescence during age-related bone loss.

For more than 100 years, cells have been known to have a transmembrane potential, ${ }^{18}$ which is maintained by the balance between ions on both sides of the plasma membrane. Whereas early studies on membrane potential primarily focused on excitability, scientists have recently shown that dynamic membrane potential also exists in most nonexcitable cells, although its role is unclear. Emerging studies indicate that membrane potential is involved in a wide range of biological functions of cells, including the cell cycle of T cells, ${ }^{36}$ proliferation of vascular smooth muscle cells, ${ }^{37}$ volume control of chondrocytes, ${ }^{38}$ secretion of pancreatic $\beta$ cells, ${ }^{39}$ migration of epithelial cells $^{40}$, and circadian rhythm of fibroblasts. ${ }^{41}$ Lallet-Daher et al. first reported alterations of plasma membrane potential in cell senescence. $^{42}$ Thereafter, the senescence of fibroblasts ${ }^{19}$ and epithelial cells ${ }^{20}$ was ascribed to membrane potential changes in several studies, although the responsible mechanisms are 
a

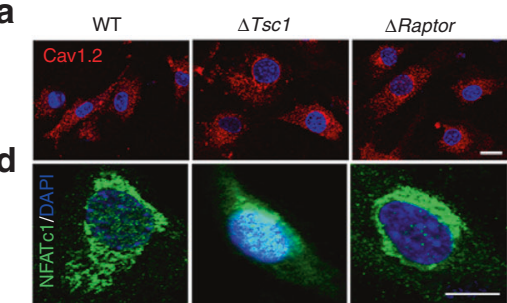

e

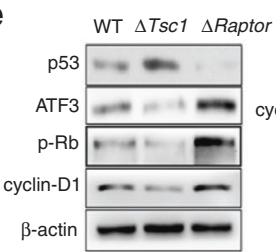

f

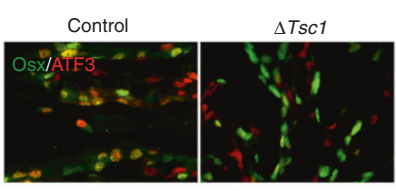

Control
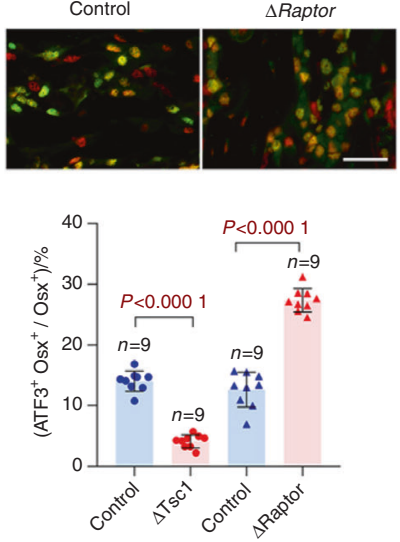

m

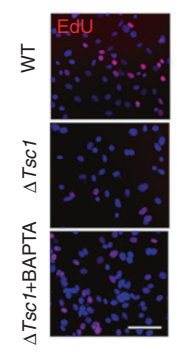

g

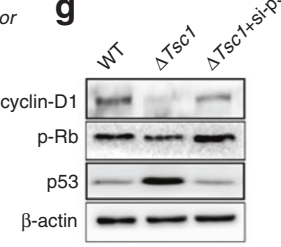

h

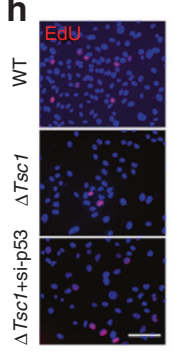

j

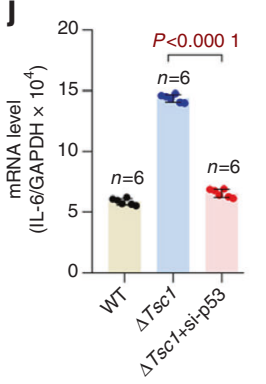

n

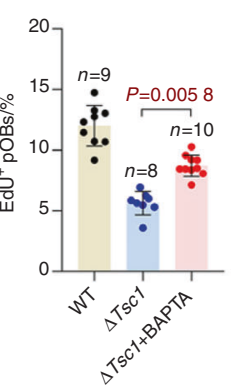

b
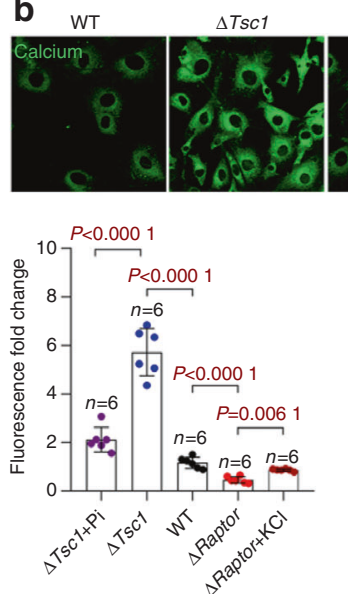

C

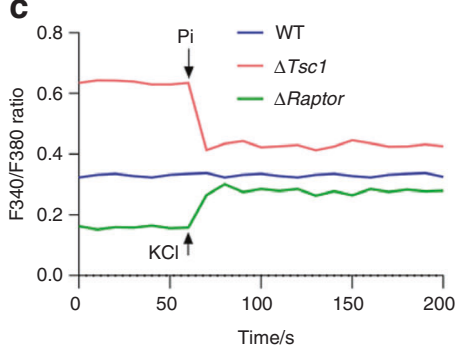

i
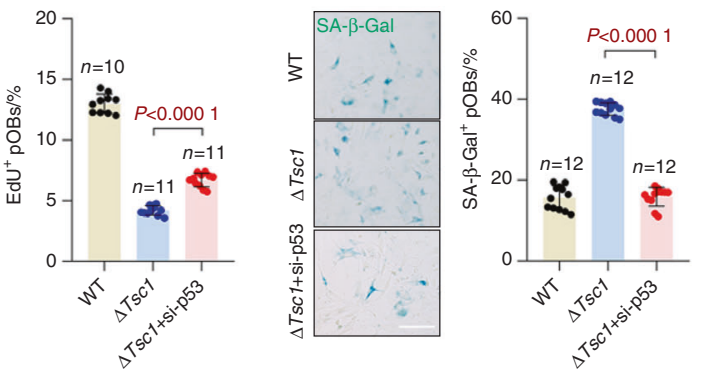

k
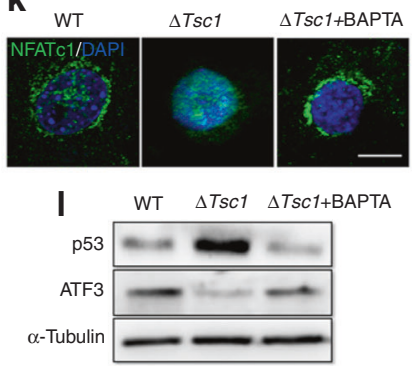

o
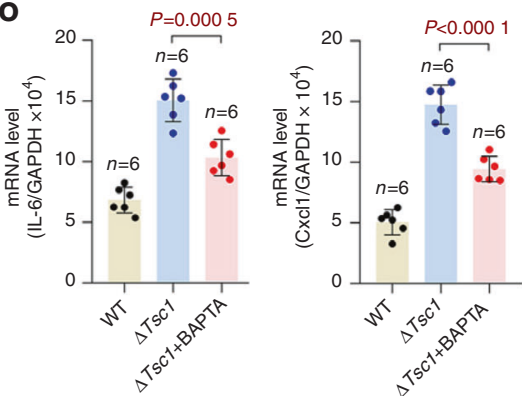

Fig. 8 Plasma membrane depolarization induces preosteoblast senescence via the $\mathrm{Ca}^{2+}$ /NFAT/ATF3/p53 signaling pathway. a Representative images of immunostaining of the voltage-gated calcium channel $\mathrm{Ca}_{v} 1.2$ in primary preosteoblasts. Scale bar, $20 \mu \mathrm{m}$. $\mathbf{b}$ Senescent $\Delta T s c 1$ and $\Delta$ Raptor preosteoblasts were treated with pinacidil $(\mathrm{Pi})$ or $\mathrm{KCl}$ and incubated with the FluoForte probe to measure the relative cytosolic calcium levels. Calcium imaging data were quantified by normalization of the values to those of senescent wild-type (WT) preosteoblasts. Scale bar, $20 \mu \mathrm{m}$. c Representative $\mathrm{Ca}^{2+}$ traces for the senescent $\Delta T s C 1$ and $\Delta$ Raptor preosteoblasts with added pinacidil or KCl. d Immunostaining of NFATc1 and (e) western blot analysis of ATF3, p53, cyclin D1, and Rb phosphorylation levels in the senescent $\Delta$ Tsc1 and $\Delta$ Raptor preosteoblasts. Scale bar, $10 \mu \mathrm{m}$ in $\mathbf{d}$. $\mathbf{f}$ Immunostaining of ATF3 and Osx in the $\Delta T s c 1$ and $\Delta$ Raptor mouse bone and quantitative analysis of the ATF $3^{+}$ preosteoblasts (ATF3 ${ }^{+} \mathrm{Osx}^{+}$) relative to the total Osx ${ }^{+}$preosteoblasts. Scale bar, $100 \mu \mathrm{m}$. $\mathbf{g}$ Western blot analysis of cyclin D1 expression and Rb phosphorylation in the $\Delta T s c 1$ preosteoblasts with interference with p53 expression. $\mathbf{h}$ Immunostaining of EdU in cells in $\mathbf{g}$ and quantitative analysis of EdU ${ }^{+}$cells relative to total cells. Scale bar, $100 \mu \mathrm{m}$. i SA- $\beta-$ gal staining of the cells in $\mathrm{g}$ and quantification of the proportion of SA- $\beta-$ galpositive cells in each population. Scale bar, $100 \mu \mathrm{m}$. j qPCR analysis of Cxcl1 and IL-6 mRNA in the cells in $\mathbf{g}$. $\mathbf{k}$ Immunostaining of NFATc1 in the senescent $\triangle T s c 1$ preosteoblasts treated with BAPTA-AM (a calcium chelator) or left untreated. Scale bar, $10 \mu \mathrm{m}$. I Western blot analysis of ATF3 and p53 expression in the cells in $\mathbf{k}$. $\mathbf{m}$ Immunostaining of EdU in the cells in $\mathbf{k}$ and quantitative analysis of EdU ${ }^{+}$cells relative to total cells. Scale bar, $100 \mu \mathrm{m}$. $\mathbf{n}$ SA- $\beta$-gal staining of the cells in $\mathbf{k}$ and quantification of the proportion of SA- $\beta$-gal-positive cells in each population. Scale bar, $100 \mu \mathrm{m}$. o qPCR analysis of Cxcl1 and IL- 6 mRNA in the cells in $\mathbf{k}$. Data are shown as the mean \pm SD. The numbers of samples ( $n$ ) are indicated in each figure panel. $P$ values were determined by two-tailed Student's $t$ test for single comparisons 


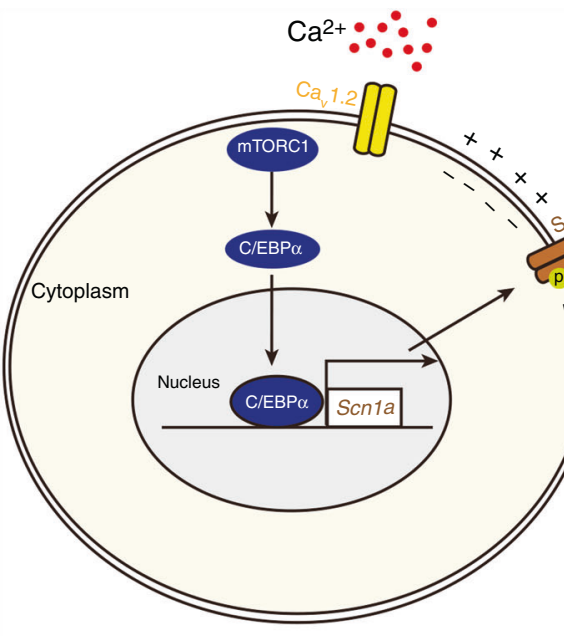

Replicative preosteoblast

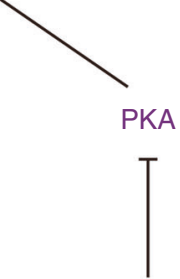

Pro-senescent stress

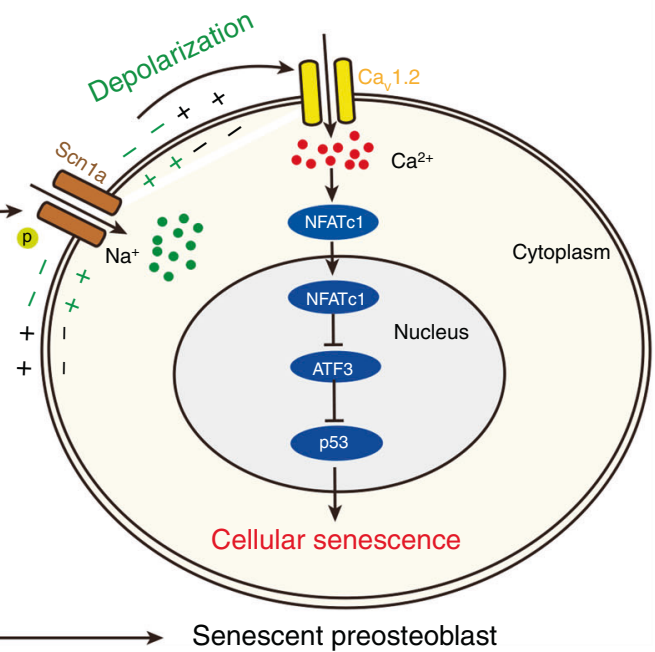

Fig. 9 Model of the sodium channel Scn1a in regulating preosteoblast senescence. Scn 1 a expression is positively regulated by mTORC1 upstream of $C / E B P \alpha$, whereas its permeability to $\mathrm{Na}^{+}$is gated by protein kinase $A$ (PKA)-induced phosphorylation. Prosenescent stresses increase the permeability of Scn 1 a to $\mathrm{Na}^{+}$by suppressing PKA activity and induce depolarization in preosteoblasts. Depolarization increases $\mathrm{Ca}^{2+}$ influx and activates downstream NFAT/ATF3/p53 signaling, thus promoting preosteoblast senescence

unclear. Here, we showed that depolarization induces preosteoblast senescence by increasing $\mathrm{Ca}^{2+}$ influx and activating downstream NFAT/ATF3/p53 signaling.

Voltage-gated calcium $\left(\mathrm{Ca}^{2+}\right)$ channels (VGCCs) are key transducers of membrane potential changes into intracellular $\mathrm{Ca}^{2+}$ transients. ${ }^{43}$ We found that $\mathrm{Ca}_{\mathrm{v}} 1.2$ is constitutively expressed by preosteoblasts and might mediate the $\mathrm{Ca}^{2+}$ influx caused by depolarization. Moreover, Fei et al. found that $\mathrm{Ca}_{\mathrm{v}} 1.2$ per se facilitates the osteogenesis of bone marrow-derived mesenchymal stem cells, and upregulation of $\mathrm{Ca}_{\mathrm{v}} 1.2$ expression alleviated osteoporosis in premature aging mice. ${ }^{44}$ However, $\mathrm{Ca}_{\mathrm{v}} 1.2$ exhibited consistent expression among the two sets of transgenic preosteoblasts and the wild-type controls in the present study. Therefore, the increased $\mathrm{Ca}^{2+}$ influx in the $\Delta T s c 1$ preosteoblasts was due to elevated $\mathrm{Ca}^{2+}$ channel activation after membrane depolarization in the cells.

Beyond increasing $\mathrm{Ca}^{2+}$ influx, depolarization might exert other adverse effects on senescent cells. For example, depolarization elevates intracellular $\mathrm{Na}^{+}$, which compromises $\mathrm{Na}^{+}$-dependent nutrient transport into cells. Coincidentally, senescent cells have been reported to be defective in nutrient sensing. ${ }^{19}$ Given the various adverse effects on cells and the possibility of reversible and rapid modulation, the membrane potential of osteoblasts is a promising target for the development of safe and rapid response drugs for the treatment of age-related bone loss.

The present study revealed that the sodium channel Scn1a mediates membrane depolarization in senescent preosteoblasts. The Scn1a gene encodes the a subunit of the voltage-gated sodium transmembrane protein $\mathrm{Na}_{\mathrm{v}} 1.1$, which allows sodium ions to flow into cells, causes membrane depolarization and induces action potentials in excitable cells. ${ }^{45} \mathrm{Na}_{\mathrm{v}} 1.1$ consists of an a subunit and two $\beta$ subunits. ${ }^{46}$ In the present study, the expression of the a subunit ( $\operatorname{Scn} 1 \mathrm{a})$, but not the $\beta$ subunits, was revealed to be regulated by $\mathrm{mTORC1}$ during preosteoblast senescence. Moreover, knockdown of $\mathrm{Scn} 1 \mathrm{a}$ was sufficient to alleviate membrane depolarization in the senescent $\Delta T s c 1$ preosteoblasts. On the basis of these observations, we inferred that the a subunit alone was sufficient to induce sodium channel function in preosteoblasts. In support of this possibility, the Na-channel a subunit has been found to act as a channel in oocytes independently of the $\beta$ subunits. ${ }^{47-49}$

Despite the important roles of Scn1a in membrane depolarization and related biological processes, little is currently known about the machinery underlying the regulation of Scn1a transcription. Voltage-gated sodium channels generally have complex $5^{\prime}$ UTRs consisting of multiple alternatively spliced noncoding exons distributed over large genomic intervals. ${ }^{23}$ Among the sodium channels, SCN1A has the most complex $5^{\prime}$ UTR organization, with seven noncoding exons distributed over a $75-\mathrm{kb}$ interval upstream of exon $1 .^{50}$ These $5^{\prime}$-UEs, with 70 putative transcription factor-binding sites, greatly enhance Scn1a gene expression. $^{25}$ In the present study, we found that Scn1a transcription was positively regulated by mTORC1. Because C/ EBPa is known to act downstream of $m$ TORC $1^{24,51}$ and has been predicted to bind the $5^{\prime}$-UE of $\operatorname{Scn} 1 a^{25}$ we further investigated whether $\mathrm{C} / \mathrm{EBPa}$ might mediate the positive regulation of Scn1a by $\mathrm{mTORC} 1$. Indeed, we observed binding of C/EBPa to the $5^{\prime}$-UE of Scn1a in osteoblasts, and this binding was promoted by mTORC1 activation but attenuated by mTORC1 inhibition. In addition, downregulation of $\mathrm{C} / \mathrm{EBPa}$ expression reversed the increased expression of Scn1a by mTORC1 activation, thus clearly demonstrating that $\mathrm{C} / \mathrm{EBPa}$ mediates the positive regulation of Scn 1 a expression by mTORC1.

mTORC1 is well known for its beneficial roles in promoting development and growth. ${ }^{52,53}$ Later in life, however, mTORC1 exerts detrimental effects by driving senescence and increasing the risk of disease. ${ }^{15}$ The age-specific roles of mTORC1 conform well to the antagonistic pleiotropy hypothesis, which suggests that aging is a byproduct of an investment in development and reproduction and that genetic variants favored in the fertile stages can cause senescence later in life. ${ }^{54,55}$ mTORC1 also appears to exert age-specific effects on bone. We previously revealed a positive effect of mTORC1 on preosteoblast expansion and bone formation during bone development, ${ }^{16,17}$ whereas the present study further uncovered the adverse effects of mTORC1 on preosteoblasts and bone mass maintenance in old age.

mTORC1-driven cell hyperproliferation and anabolism are generally presumed to cause aging. ${ }^{56}$ However, we found that mTORC1 activation-induced preosteoblast senescence does not require mTORC1-driven overgrowth in earlier stages because mTORC1 was not activated at the early ages in the mice in the present study. Instead, the distinct roles of mTORC1 on osteoblast and bone formation in old age are ascribed to the unique membrane depolarization in senescent preosteoblasts. mTORC 1 controls membrane potential by regulating the expression of the sodium channel Scn1a; however, the opening 
probability of Scn1a remains inhibited by PKA-induced phosphorylation. ${ }^{26,57}$ The increased Scn 1 a by mTORC1 activation does not evoke depolarization in the replicative $\Delta T s c 1$ preosteoblasts during bone development because most Scn1a channels are likely to be closed owing to phosphorylation by excess PKA activity. In the preosteoblasts of aged mice, however, most Scn 1 a channels are open, thus causing depolarization and inducing cell senescence in the presence of the decreased PKA activity resulting from prosenescent stress. ${ }^{29}$ Consistently, Lallet-Daher et al. also demonstrated decreased PKA activity in senescent epithelial cells, which induces cell senescence by activating the potassium channel KCNA1 and altering the membrane potential. ${ }^{42}$ PKA appears to exert central roles in the membrane potential changes during cell senescence by gating control of ion channels, thus providing an explanation for the age-specific effects of mTORC1 on preosteoblasts and shedding new light on the antagonistic pleiotropy of mTORC1.

In conclusion, we reveal the mechanisms underlying mTORC1/ Scn1a/plasma membrane depolarization-induced osteoblast senescence, including the transcriptional regulation of Scn1a by mTORC1, gating control of Scn1a by prosenescent stress, and the involvement of Scn1a, plasma membrane depolarization and calcium in the regulation of the NFAT/ATF3/p53 pathway. Hence, this work provides new insights into the involvement of ion channels and plasma membrane potential in the control of preosteoblast senescence. Pharmaceutical studies of the related pathways and agents might lead to novel potential treatments for age-related bone loss.

\section{MATERIALS AND METHODS}

Mice

Transgenic Osx-cre [006361-B6.Cg-Tg(Sp7-tTA,tetO-EGFP/cre) $1 \mathrm{Amc} / \mathrm{J}], T s c 1^{\text {flox/flox }}\left(005680-\mathrm{STOCK} T s \mathrm{~T} 1^{\text {tmiDjk }} / \mathrm{J}\right)$ and Raptor ${ }^{\text {flox/flox }}$ (013188-B6.Cg-Rptor ${ }^{\text {tm1.1Dmsa } / J) ~ m o u s e ~ s t r a i n s ~ w e r e ~ p u r c h a s e d ~}$ from the Jackson Laboratory. The background of the $T s \mathrm{Cl}^{\text {flox/flox }}$ mice was $129 \mathrm{~s} 4 / \mathrm{SvJae}$, and these mice were backcrossed to a C57BL/6 background for eight generations before use. Genotyping of these mice was conducted with genomic DNA isolated from tail biopsies, with the primers listed in Table S1. To prevent the Osx promoter from driving Cre expression, we exposed the mice to $200 \mu \mathrm{g} \cdot \mathrm{mL}^{-1}$ doxycycline (Sigma-Aldrich) in their drinking water. C57BL/6J (wild-type) mice were purchased from the Experimental Animal Center of Southern Medical University (Guangzhou, China).

\section{Micro-CT analysis}

The three-dimensional structure of trabecular bone was analyzed in the femora or tibias on a micro-CT scanner (Viva CT40; Scanco Medical AG, Bassersdorf, Switzerland). For the femora, scanning was started from the lower growth plate and extended proximally for 300 slices at $12-\mu \mathrm{mol} \cdot \mathrm{L}^{-1}$ resolution. Morphometric analysis was conducted with the first slice in which the femoral condyles were fully merged and extended for 100 slices proximally. For tibias, scanning was performed in the proximal metaphysis, starting from an anatomic landmark in the growth plate and extending 300 slices distally.

Morphometric analysis was conducted with the first slice in which the growth plate disappeared and was extended for 100 slices distally. The trabecular bones of the femora and tibias were segmented from the cortical shell manually on key slices by using a contouring tool, and then, the contours were morphed automatically to segment the trabecular bone on all slices. The three-dimensional structure and morphometry were constructed and analyzed for trabecular bone volume fraction (BV/TV), trabecular thickness (Tb.Th), trabecular number (Tb.N) and trabecular separation (Tb.Sp). Two-dimensional images were used to generate $3 \mathrm{D}$ reconstructions in the image processing software Materialize Mimics (Materialize NV, Belgian).
Immunohistochemistry and histochemistry staining

Mouse tibial tissues were fixed with $4 \%$ paraformaldehyde in PBS at $4{ }^{\circ} \mathrm{C}$ for $48 \mathrm{~h}$ and then $10 \%$ EDTA ( $\mathrm{pH} \mathrm{8.0)}$ ) at $4{ }^{\circ} \mathrm{C}$ for 14 days. The tissues were embedded in paraffin or optimal cutting temperature compound (Sakura Finetek), and 3-5 $\mu \mathrm{m}$ sagittally oriented sections were prepared for histological analyses. For immunohistochemistry, we incubated primary antibodies that recognized mouse phospho-S6 (Ser235/236) (Cell Signaling, 1:100, \#2211), Osterix (Abcam, 1:200, ab22552), osteocalcin (Abcam, \#ab93876), p16 (Santa Cruz Biotechnology, 1:200, \#sc1661) and Scn1a (Boster Biological Technology, 1:200, \#PB0932) overnight at $4{ }^{\circ} \mathrm{C}$. TRAP staining (Sigma-Aldrich, Missouri, USA) was performed according to the manufacturer's instructions. Cells per bone perimeter (B.Pm) or the percentage of positive cells was used to calculate the number of positive cells. At least three mice per group were examined. Three equidistant sections spaced $200 \mu \mathrm{m}$ apart throughout the midsagittal sections of the tibia were evaluated.

\section{Cell culture and treatment}

We purchased the mouse preosteoblast cell line MC3T3-E1 from the American Type Culture Collection. The cells were maintained in a-minimal essential Eagle's medium (aMEM, Corning) containing $10 \% \mathrm{FBS}$ (Gibco) at $37^{\circ} \mathrm{C}$ with $5 \% \mathrm{CO}_{2}$. Calvarial osteoblastic cells were prepared from the calvariae of newborn mice $(24 \mathrm{~h}$ after birth), washed with PBS and digested in $0.1 \mathrm{mg} \cdot \mathrm{mL}^{-1}$ collagenase type II (Thermo Fisher Scientific, \#17101015) in aMEM at $37^{\circ} \mathrm{C}$ for $20 \mathrm{~min}$ with five replicates. The supernatants were then combined and centrifuged to pellet the cells after digestion. We also isolated osteoblastic cells from the long bones of mice as described previously. ${ }^{58}$ Primary osteoblastic cells were cultured in aMEM supplemented with $10 \%$ FBS and $100 \mathrm{U} \cdot \mathrm{mL}^{-1}$ penicillin/streptomycin (Invitrogen) and then incubated with tert-butyl hydroperoxide (t-BHP) (Sigma-Aldrich, $100 \mu \mathrm{mol} \cdot \mathrm{L}^{-1}$ ), pinacidil (MedChemExpress, $100 \mu \mathrm{mol} \cdot \mathrm{L}^{-1}$, \#HY-14290A), H-89 (Beyotime Institute of Biotechnology, $10 \mu \mathrm{mol} \cdot \mathrm{L}^{-1}$, \#S1643), forskolin (Meilunbio, $10 \mu \mathrm{mol} \cdot \mathrm{L}^{-1}$, \#MB5959), IBMX (Meilunbio, $100 \mu \mathrm{mol} \cdot \mathrm{L}^{-1}$, \#MB5226) or BAPTA-AM (MedChemExpress, $\left.20 \mu \mathrm{mol} \cdot \mathrm{L}^{-1}, \# \mathrm{HY}-100545\right)$ treatment as indicated.

For ROS-induced cell senescence, preosteoblasts were incubated with tert-butyl hydroperoxide (t-BHP) (Sigma-Aldrich, $\left.100 \mu \mathrm{mol} \cdot \mathrm{L}^{-1}, \# 458139\right)$ for three consecutive days. To induce osteogenic differentiation, we plated primary osteoblastic cells at a density of $1.5 \times 10^{5}$ cells/well (six-well plates) and incubated them with osteoblast differentiation medium with $\beta$-glycerophosphate (Sigma-Aldrich, $5 \mathrm{mmol} \cdot \mathrm{L}^{-1}$ ) and ascorbic acid (Sigma-Aldrich, $50 \mu \mathrm{g} \cdot \mathrm{mL}^{-1}$ ).

\section{Cell staining}

For SA- $\beta$-galactosidase staining, cells were washed with PBS, fixed with 4\% paraformaldehyde in PBS at room temperature for 20 min and incubated with reagents from a senescence-associated $\beta$-galactosidase staining kit (Beyotime Institute of Biotechnology, \#C0602) according to the manufacturer's suggestions.

For alkaline phosphatase staining, differentiated osteoblasts were washed with PBS, fixed with $4 \%$ paraformaldehyde for $30 \mathrm{~min}$ at room temperature and stained with an Alkaline Phosphatase Staining Kit (Beyotime Institute of Biotechnology) for $1 \mathrm{~h}$ at room temperature in the dark.

For alizarin red staining, cells were fixed with paraformaldehyde for $30 \mathrm{~min}$, incubated with $1 \%$ alizarin red for $30 \mathrm{~min}$ at room temperature and washed with PBS to remove the excess dye.

For immunocytochemical staining, we incubated cultured cells with primary antibodies recognizing mouse $\mathrm{Ca}_{\mathrm{v}} 1.2$ (Abbkine, 1:100, \#Abp57305) or NFATc1 (Santa Cruz Biotechnology, 1:100, \#sc-7294) overnight at $4{ }^{\circ} \mathrm{C}$. Secondary antibodies conjugated with fluorescent tags were incubated at room temperature for $1 \mathrm{~h}$ in the dark. 
siRNA infection

The siRNAs targeting mouse $S c n 1 a$ and C/EBPa were designed and synthesized by GenePharma Co., Ltd. (Shanghai, China). The sequences of Scn $1 \mathrm{a}$ and C/EBPa siRNA were as follows: siScn1a， 5'-GCCUGUCAUUGAACCAGAATT-3'; si-C/EBPa， 5'-GAG CCGAGAUAAAGCCAAATT- $3^{\prime}$. Adenovirus encoding siRNA targeting mouse Scn 1 a was produced by Dongze Biotech Co., Ltd. (Guangzhou, China).

We transiently transfected cells with siRNA by using Lipofectamine 3000 (Invitrogen, Carlsbad, CA, USA) in Opti-MEM (Invitrogen) according to the manufacturer's instructions. The efficiency of transfection was measured by western blotting. Adenoviruses $\left(2 \times 10^{9}\right.$ PFU per mouse) were applied to mice once every 3 days for 1 month via tail-vein injection.

\section{Western blotting}

Cell lysis buffer containing 2\% SDS, $2 \mathrm{~mol} \cdot \mathrm{L}^{-1}$ urea, $10 \%$ glycerol, $10 \mathrm{mmol} \cdot \mathrm{L}^{-1}$ Tris- $\mathrm{HCl}\left(\mathrm{pH}\right.$ 6.8), $10 \mathrm{mmol} \cdot \mathrm{L}^{-1}$ dithiothreitol and $1 \mathrm{mmol} \cdot \mathrm{L}^{-1}$ phenylmethylsulfonyl fluoride was added to lyse adherent cells. The lysates were centrifuged, and the supernatants were separated by SDS-polyacrylamide gel electrophoresis and blotted onto a PVDF membrane (Merck Millipore, ISEQ00010). The membrane was then incubated with specific antibodies against phospho-S6 (S235/236) (Cell Signaling Technology, 1:1 000, \#2211), S6 (Santa Cruz Biotechnology, 1:4 000, \#sc-74459), p16 (Santa Cruz Biotechnology, 1:2 000, sc-1661), ATF3 (Abbkine, 1:1 000, \#Abp55330), p53 (Cell Signaling Technology, 1:2 000, \#2524), Scn1a (Boster Biological Technology, 1:1 000, \#PB0932) and C/EBPa (Cell Signaling Technology, 1:2 000, \#2295). The membrane was then visualized by enhanced chemiluminescence (ECL Kit, Amersham Biosciences).

Real-time quantitative PCR

Total RNA was isolated from cell pellets with TRIzol Reagent (TaKaRa Biotechnology, \#9109). cDNA was reverse transcribed from RNA samples with reverse transcription reagents (TaKaRa Biotechnology, \#RR036A), and qPCR assays were performed with Real-Time PCR Mix (Vazyme Biotech, \#Q311-02) in a Light Cycler (Roche Molecular Biochemicals, Indianapolis, IN, USA). Primer sequences are listed in Table S1. The relative quantification of gene expression was performed with the comparative threshold $\left(C_{T}\right)$ method. Changes in mRNA expression levels were calculated after normalization to values for the GAPDH calibrator gene.

Cell proliferation assays

A kFluor555-EdU cell proliferation detection kit (Keygen Biotechnology, Jiangsu, China; \#KGA337) was used to detect cell proliferation according to the manufacturer's instructions. Briefly, cells were seeded in 96-well plates (6 000 cells/well) and incubated with $50 \mathrm{\mu mol} \cdot \mathrm{L}^{-1} \mathrm{EdU}$ at $37^{\circ} \mathrm{C}$ and $5 \% \mathrm{CO}_{2}$ for $3 \mathrm{~h}$. After fixation with $4 \%$ paraformaldehyde for $20 \mathrm{~min}$ at room temperature, the cells were stained with a kFluor555 reaction mixture for immunocytochemical analysis. Nine areas in each group were counted by two independent observers blinded to the groups.

Chromatin immunoprecipitation (ChIP) assays

ChIP assays were conducted with a ChIP assay kit (Cell Signaling Technology) according to the manufacturer's instructions. Approximately $2 \times 10^{7}$ cells were incubated with $1 \%$ formaldehyde to crosslink the protein and DNA. After a 10-min incubation, a final concentration of $0.125 \mathrm{~mol} \cdot \mathrm{L}^{-1}$ glycine was added to the $1 \%$ formaldehyde-PBS solution for neutralization. The harvested cells were lysed, and genomic DNA was sonicated to decrease the DNA length to 400 to $800 \mathrm{bp}$. The sheared chromatin was immunoprecipitated with $4 \mu \mathrm{g}$ anti-C/EBPa (Proteintech, 18311-1$\mathrm{AP}$ ) or normal IgG as a negative control. Isolated DNA was subjected to PCR both before and after chromatin immunoprecipitation with primers designed to amplify the region within the Scn1a 5'-UE containing the C/EBPa binding site. The primers are listed in Table S1. The PCR products were assessed on $1.0 \%$ agarose gels and confirmed by direct sequencing. qPCR was performed according to the above description.

Plasma membrane potential measurement

Plated cells were washed with PBS and incubated with $200 \mathrm{nmol} \cdot \mathrm{L}^{-1}$ of the membrane voltage-reporting dye DiBAC4 (Molecular Probes) in PBS at $37^{\circ} \mathrm{C}$ for $1 \mathrm{~h}$. Then, the cells were observed and photographed with a FluoView FV1000 confocal microscope (Olympus). The fluorescence data were analyzed with Image-Pro Plus software.

Alternatively, plasma membrane potential was evaluated with the conventional whole-cell configuration of the patch-clamp technique at room temperature. The internal solution $(\mathrm{pH}=7.2)$ contained $130 \mathrm{mmol} \cdot \mathrm{L}^{-1} \mathrm{KCl}, 1 \mathrm{mmol} \cdot \mathrm{L}^{-1} \mathrm{MgCl}_{2}, 3 \mathrm{mmol} \cdot \mathrm{L}^{-1}$ MgATP and $5 \mathrm{mmol} \cdot \mathrm{L}^{-1}$ HEPES. The external solution $(\mathrm{pH}=7.4)$ contained $140 \mathrm{mmol} \cdot \mathrm{L}^{-1} \mathrm{NaCl}, 4 \mathrm{mmol} \cdot \mathrm{L}^{-1} \mathrm{KCl}, 2 \mathrm{mmol} \cdot \mathrm{L}^{-1} \mathrm{MgCl}_{2}$, $2.5 \mathrm{mmol} \cdot \mathrm{L}^{-1} \mathrm{CaCl}_{2}, 10 \mathrm{mmol} \cdot \mathrm{L}^{-1}$ glucose and $10 \mathrm{mmol} \cdot \mathrm{L}^{-1} \mathrm{HEPES}$. Membrane currents were evoked from a holding potential of $-80 \mathrm{mV}$ by voltage ramps of 1.5 -s duration, applied from -100 to $+90 \mathrm{mV}$, sampled at $1 \mathrm{kHz}$, and filtered at $300 \mathrm{~Hz}$. Reversal potentials of ramp membrane currents were determined by linear fits extrapolated on-ramp currents in a potential range around the zero current and were verified in the 10 current-clamp mode of the patch-clamp amplifier to be close to the membrane potentials of cells.

Cytosolic calcium level measurements and PKA enzymatic activity assays

Cytosolic calcium levels were detected with a FluoForte Calcium Assay Kit (Enzo Life Sciences, \#ENZ-51017) and Fura-2/AM (Invitrogen ${ }^{\mathrm{TM}}$, \# F1221) according to the manufacturer's suggested procedure. Briefly, cells plated in 96-well plates (40 000 cells/well) were incubated with $100 \mu \mathrm{L}$ of FluoForte Dye-loading solution at $37^{\circ} \mathrm{C}$ for $1 \mathrm{~h}$. Then, the cells were photographed with a FluoView FV1000 confocal microscope (Olympus). Fluorescence micrographs were digitalized, and the results are expressed as the change in fluorescence over baseline fluorescence.

Preosteoblasts were further loaded with $5 \mu \mathrm{mol} \cdot \mathrm{L}^{-1}$ Fura-2/AM in Hanks' balanced salt solution (HBSS) for $1 \mathrm{~h}$ at $37^{\circ} \mathrm{C}$. After the cells were washed extensively with HBSS, cytosolic $\mathrm{Ca}^{2+}$ was measured with a calcium imaging system built on an inverted fluorescence microscope (Olympus IX51). Fluorescence images (filtered at $515 \mathrm{~nm} \pm 25 \mathrm{~nm}$ ) were captured with a CCD camera (CoolSNAP fx-M) and analyzed in MetaFluor software. $\mathrm{Ca}^{2+}$ levels are shown as the ratio of fluorescence intensity at $340 \mathrm{~nm} /$ fluorescence intensity at $380 \mathrm{~nm}$ (F340/F380). At least three independent experiments were performed for each condition.

Total cellular and mitochondrial PKA activities were measured with a nonradioactive PKA Kinase Activity Assay Kit (\#ab139435, Abcam) according to the manufacturer's instructions. ${ }^{59}$

Statistical analysis

All results are presented as the mean \pm SD. Curve analysis was performed in Prism (GraphPad). The data in each group were analyzed with unpaired, two-tailed Student's $t$ test. The significance threshold was set at $P<0.05$.

\section{DATA AVAILABILITY}

The authors declare that all data supporting the findings of this study are available. within the article and its Supplementary Information files or from the corresponding author upon reasonable request. The global mRNA expression profile reported in this study has been deposited in the Gene Expression Omnibus (GEO) database under the accession code GSE74781. 


\section{ACKNOWLEDGEMENTS}

This research was supported by grants 82172507 (B.H.), 81700783 (B.H.), and 81672120 (D.J.) from the National Natural Science Foundation of China, 2019A1515011876 (B.H.) and 2018A030313937 (Z.L.) from the Guangdong Natural Science Fund Management Committee and 202002030176 (B.H.) from the Guangzhou Municipal Science and Technology Bureau.

\section{AUTHOR CONTRIBUTIONS}

B.H. conceived the ideas for the experimental design, analyzed the data, and wrote the paper; A.C. and J.J. conducted the majority of the experiments and helped with the paper preparation; Z.L. and C.Y. performed the microcomputed tomography analyses; Q.C. and W.L. performed the immunohistochemistry and immunofluorescence and confocal imaging; S.C., K.L., and D.K. conducted the cell cultures and western blot experiments; Z.O. and C.Y. maintained the mice and collected the tissue samples; X.B., Q.L., and D.J. developed the concept, supervised the project, conceived the experiments, and critically reviewed the paper.

\section{ADDITIONAL INFORMATION}

Supplementary information The online version contains supplementary material available at https://doi.org/10.1038/s41413-022-00204-1.

Competing interests: The authors declare no competing interests.

\section{REFERENCES}

1. Owen, R. \& Reilly, G. C. In vitro models of bone remodelling and associated disorders. Front. Bioeng. Biotechnol. 6, 134 (2018).

2. Farr, J. N. et al. Identification of senescent cells in the bone microenvironment. J. Bone Miner. Res. 31, 1920-1929 (2016).

3. Farr, J. N. et al. Targeting cellular senescence prevents age-related bone loss in mice. Nat. Med. 23, 1072-1079 (2017).

4. Manolagas, S. C. \& Jilka, R. L. Bone marrow, cytokines, and bone remodeling. Emerging insights into the pathophysiology of osteoporosis. N. Engl. J. Med. 332, 305-311 (1995).

5. Kassem, M. \& Marie, P. J. Senescence-associated intrinsic mechanisms of osteoblast dysfunctions. Aging Cell 10, 191-197 (2011).

6. Manolagas, S. C. \& Parfitt, A. M. What old means to bone. Trends Endocrinol. Metab. 21, 369-374 (2010).

7. Long, F. Building strong bones: molecular regulation of the osteoblast lineage. Nat. Rev. Mol. Cell Biol. 13, 27-38 (2011).

8. Kim, H. N. et al. DNA damage and senescence in osteoprogenitors expressing Osx1 may cause their decrease with age. Aging Cell 16, 693-703 (2017).

9. Cao, J. J. et al. Aging impairs IGF-I receptor activation and induces skeletal resistance to IGF-I. J. Bone Miner. Res. 22, 1271-1279 (2007).

10. Pfeilschifter, J. et al. Mitogenic responsiveness of human bone cells in vitro to hormones and growth factors decreases with age. J. Bone Min. Res. 8, 707-717 (1993).

11. Ankrom, M. A. et al. Age-related changes in human oestrogen receptor alpha function and levels in osteoblasts. Biochem. J. 333, 787-794 (1998).

12. Undale, A. et al. Circulating osteogenic cells: characterization and relationship to rates of bone loss in postmenopausal women. Bone 47, 83-92 (2010).

13. Papadopoli, D. et al. mTOR as a central regulator of lifespan and aging. F1000Res. 8, F1000 Faculty Rev_998 (2019).

14. Guertin, D. A. et al. Ablation in mice of the mTORC components raptor, rictor, or $\mathrm{mLST} 8$ reveals that $\mathrm{mTORC2}$ is required for signaling to Akt-FOXO and $\mathrm{PKCa}$, but Not S6K1. Dev. Cell 11, 859-871 (2006).

15. Kapahi, P. et al. With TOR, less is more: a key role for the conserved nutrientsensing TOR pathway in aging. Cell Metab. 11, 453-465 (2010).

16. Huang, B. et al. mTORC1 prevents preosteoblast differentiation through the notch signaling pathway. PLoS Genet. 11, e1005426 (2015).

17. Huang, B. et al. Osteoblasts secrete Cxcl9 to regulate angiogenesis in bone. Nat. Commun. 7, 13885 (2016).

18. Höber, R. Über den Einfluss der Salze auf den Ruhestrom des Froschmuskels. Arch. $f$. ür. die Gesamt. Physiologie des. Menschen und der Tiere 106, 599-635 (1905).

19. Carroll, B. et al. Persistent mTORC1 signaling in cell senescence results from defects in amino acid and growth factor sensing. J. Cell Biol. 216, 1949-1957 (2017).

20. Warnier, M. et al. The SCN9A channel and plasma membrane depolarization promote cellular senescence through Rb pathway. Aging Cell 17, e12736 (2018).

21. Chen, J. \& Long, F. mTORC1 signaling promotes osteoblast differentiation from preosteoblasts. PloS ONE 10, e0130627-e0130627 (2015).

22. Fitter, S. et al. mTORC1 plays an important role in skeletal development by controlling preosteoblast differentiation. Mol. Cell. Biol. 37, e00668-00616 (2017).

23. Catterall, W. A. Forty years of sodium channels: structure, function, pharmacology, and epilepsy. Neurochem. Res. 42, 2495-2504 (2017).

24. Laplante, M. \& Sabatini, D. M. An emerging role of mTOR in lipid biosynthesis. Curr. Biol. 19, R1046-R1052 (2009).

25. Long, Y. S. et al. Identification of the promoter region and the $5^{\prime}$-untranslated exons of the human voltage-gated sodium channel Nav1.1 gene (SCN1A) and enhancement of gene expression by the $5^{\prime}$-untranslated exons. J. Neurosci. Res. 86, 3375-3381 (2008).

26. Smith, R. D. \& Goldin, A. L. Phosphorylation at a single site in the rat brain sodium channel is necessary and sufficient for current reduction by protein Kinase A. J. Neurosci. 17, 6086-6093 (1997).

27. Smith, R. D. \& Goldin, A. L. Functional analysis of the rat I sodium channel in xenopus oocytes. J. Neurosci. 18, 811-820 (1998).

28. Liu, S. \& Zheng, P. Altered PKA modulation in the $\mathrm{Na}(\mathrm{v}) 1.1$ epilepsy variant I1656M. J. Neurophysiol. 110, 2090-2098 (2013).

29. Jin, B. et al. PIM-1 modulates cellular senescence and links IL-6 signaling to heterochromatin formation. Aging Cell 13, 879-889 (2014).

30. Courtney, M. J., Lambert, J. J. \& Nicholls, D. G. The interactions between plasma membrane depolarization and glutamate receptor activation in the regulation of cytoplasmic free calcium in cultured cerebellar granule cells. J. Neurosci. 10, 3873-3879 (1990).

31. Wiel, C. et al. Endoplasmic reticulum calcium release through ITPR2 channels leads to mitochondrial calcium accumulation and senescence. Nat. Commun. 5, 3792 (2014).

32. Martin, N. \& Bernard, D. Calcium signaling and cellular senescence. Cell Calcium 70, 16-23 (2018).

33. Hogan, P. G., Chen, L., Nardone, J. \& Rao, A. Transcriptional regulation by calcium, calcineurin, and NFAT. Genes Dev. 17, 2205-2232 (2003).

34. $\mathrm{Wu}, \mathrm{X}$. et al. Opposing roles for calcineurin and ATF3 in squamous skin cancer. Nature 465, 368-372 (2010).

35. d'Adda di Fagagna, F. Living on a break: cellular senescence as a DNA-damage response. Nat. Rev. Cancer 8, 512-522 (2008).

36. Amigorena, S., Choquet, D., Teillaud, J. L., Korn, H. \& Fridman, W. H. Ion channel blockers inhibit B cell activation at a precise stage of the $\mathrm{G} 1$ phase of the cell cycle. Possible involvement of $\mathrm{K}^{+}$channels. J. Immunol. 144, 2038-2045 (1990).

37. Nelson, M. T. \& Quayle, J. M. Physiological roles and properties of potassium channels in arterial smooth muscle. Am. J. Physiol. Cell Physiol. 268, C799-C822 (1995).

38. Lewis, R. et al. The role of the membrane potential in chondrocyte volume regulation. J. Cell. Physiol. 226, 2979-2986 (2011).

39. Rorsman, P. \& Ashcroft, F. M. Pancreatic $\beta$-cell electrical activity and insulin secretion: of mice and men. Physiol. Rev. 98, 117-214 (2018).

40. Girault, A. \& Brochiero, E. Evidence of $\mathrm{K}+$ channel function in epithelial cell migration, proliferation, and repair. Am. J. Physiol. Cell Physiol. 306, C307-C319 (2014).

41. Noguchi, T., Wang, C. W., Pan, H. \& Welsh, D. K. Fibroblast circadian rhythms of PER2 expression depend on membrane potential and intracellular calcium. Chronobiol. Int. 29, 653-664 (2012).

42. Lallet-Daher, $\mathrm{H}$. et al. Potassium channel KCNA1 modulates oncogene-induced senescence and transformation. Cancer Res. 73, 5253-5265 (2013).

43. Catterall, W. A. Voltage-gated calcium channels. Cold Spring Harb. Perspect. Biol. 3, a003947-a003947 (2011).

44. Fei, D. et al. Ca(v) 1.2 regulates osteogenesis of bone marrow-derived mesenchymal stem cells via canonical Wnt pathway in age-related osteoporosis. Aging Cell 18, e12967 (2019).

45. Escayg, A. et al. Mutations of SCN1A, encoding a neuronal sodium channel, in two families with GEFS+2. Nat. Genet. 24, 343-345 (2000)

46. Catterall, W. A., Kalume, F. \& Oakley, J. C. NaV1.1 channels and epilepsy. J. Physiol. 588, 1849-1859 (2010).

47. Sumikawa, K., Parker, I. \& Miledi, R. Partial purification and functional expression of brain mRNAs coding for neurotransmitter receptors and voltage-operated channels. Proc. Natl Acad. Sci. U.S.A. 81, 7994-7998 (1984).

48. Hirono, C. et al. Characterization of mRNA responsible for induction of functional sodium channels in Xenopus oocytes. Brain Res. 359, 57-64 (1985).

49. Goldin, A. L. et al. Messenger RNA coding for only the alpha subunit of the rat brain $\mathrm{Na}$ channel is sufficient for expression of functional channels in Xenopus oocytes. Proc. Natl Acad. Sci. U.S.A. 83, 7503-7507 (1986).

50. Martin, M. S., Tang, B., Ta, N. \& Escayg, A. Characterization of $5^{\prime}$ untranslated regions of the voltage-gated sodium channels SCN1A, SCN2A, and SCN3A and identification of cis-conserved noncoding sequences. Genomics 90, 225-235 (2007).

51. Kim, J. E. \& Chen, J. regulation of peroxisome proliferator-activated receptorgamma activity by mammalian target of rapamycin and amino acids in adipogenesis. Diabetes 53, 2748-2756 (2004).

52. Gangloff, Y. G. et al. Disruption of the mouse mTOR gene leads to early postimplantation lethality and prohibits embryonic stem cell development. Mol. Cell Biol. 24, 9508-9516 (2004)

53. Porstmann, T. et al. SREBP activity is regulated by mTORC1 and contributes to Akt-dependent cell growth. Cell Metab. 8, 224-236 (2008). 
54. Williams, G. C. Pleiotropy, natural selection, and the evolution of senescence. Evolution 11, 398-411 (1957).

55. Wachter, K. W., Evans, S. N. \& Steinsaltz, D. The age-specific force of natural selection and biodemographic walls of death. Proc. Natl Acad. Sci. U.S.A. 110 10141-10146 (2013).

56. Finkel, T. Relief with rapamycin: mTOR inhibition protects against radiationinduced mucositis. Cell Stem Cell 11, 287-288 (2012).

57. Li, M., West, J. W., Lai, Y., Scheuer, T. \& Catterall, W. A. Functional modulation of brain sodium channels by CAMP-dependent phosphorylation. Neuron $\mathbf{8}$, 1151-1159 (1992).

58. Bakker, A. D. \& Klein-Nulend, J. Osteoblast isolation from murine calvaria and long bones. Methods Mol. Biol. 816, 19-29 (2012).

59. Zhu, D., You, J., Zhao, N. \& Xu, H. Magnesium regulates endothelial barrier functions through TRPM7, MagT1, and S1P1. Adv. Sci. 6, 1901166 (2019).
Open Access This article is licensed under a Creative Commons Attribution 4.0 International License, which permits use, sharing, adaptation, distribution and reproduction in any medium or format, as long as you give appropriate credit to the original author(s) and the source, provide a link to the Creative Commons license, and indicate if changes were made. The images or other third party material in this article are included in the article's Creative Commons license, unless indicated otherwise in a credit line to the material. If material is not included in the article's Creative Commons license and your intended use is not permitted by statutory regulation or exceeds the permitted use, you will need to obtain permission directly from the copyright holder. To view a copy of this license, visit http://creativecommons. org/licenses/by/4.0/.

(c) The Author(s) 2022 